\title{
Bricolage: zwischen dialektaler und globaler Variation - Wie sich Jugendliche über Stilbasteleien sozial positionieren
}

\author{
Esther Galliker (Luzern)
}

\begin{abstract}
The article presents a sociolinguistic analysis of dialectal conversations in the German part of Switzerland. It focusses on an adolescent peer group, whose members position themselves socially using a wide range of stylistic variation. The highschool students' community situated in a rural area in the middle of the Swiss Alps co-construct their group identity deliberately combining various types of linguistic variants, styles and languages. Thus, they resort to elements of a widely spread young urban street style as well as to traditional and to some extent outdated dialectal variants simultaneously. On the one hand, it is the width of the linguistic resources which is remarkable. On the other hand, it is impressive to observe the youths' competence in combining different variants creating their new and own communicative style. The study sheds light on the subtle stylistic techniques at work and demonstrates how much knowledge about the peer group's linguistic practices, preferences and resources is necessary to interpret and understand their conversations and social contextualisations. The methodological approach to the analysis of linguistic variation is a conceptual one: the main communicative practice in the young men's talk are conceptualised by means of bricolage - a specific way of incorporating and adapting linguistic elements to create a new and individual speech style. The conceptual approach also allows for a comparison of the peer group's linguistic practices with speech styles of other young peer groups all over Europe: even if the specific resources used by the youth might differ to some extent, the way in which young urban and young rural pupils deal with linguistic variation is surprisingly similar.
\end{abstract}

\section{$1 \quad$ Einleitung}

Der folgende Text zeigt auf, was es bedeutet, in einem dialektalen Umfeld wie der Deutschschweiz authentisches Gesprächsmaterial soziolinguistisch zu untersuchen. Im Fokus steht dabei, wie sich die Mitglieder einer jungen Peergruppe über stilistische Prozesse sozial positionieren, sich also über ihren Sprachgebrauch eine Gruppenidentität schaffen. Die Gymnasiasten aus dem ländlichen Nidwalden bedienen sich in ihren Gesprächen gleichzeitig sprachlicher Elemente einer globalen Hiphop-Kultur sowie traditioneller, urtümlich markierter lokaler Dialektvarianten. Sie greifen kompetent eine grosse Breite an sprachlichen Ressourcen auf. Der Artikel zeigt, wie viel Wissen über sprachliche Praktiken und Ressourcen der Jugendlichen und ihres Umfeldes nötig sind, um die symbolischen Bezüge verstehen zu können, die in ihren Gesprächen hergestellt werden. Der Zugang zu diesen Variationsphänomenen geschieht über das Konzept der Bricolage, welches vertieft und in verschiedenen Ausprägungen vorge- 
stellt wird. Dieser konzeptuelle Zugang zu Variation dient auch als Grundlage, um sprachliches Handeln verschiedener Sprech- sowie Sprachgemeinschaften miteinander in Bezug setzen zu können und die Stilbasteleien der Jugendlichen in einen Makrokontext „Jugendsprache“ einzuordnen.

Die Analyse des folgenden Gesprächsausschnittes soll exemplarisch aufzeigen, welche Variationsphänomene in den Gesprächen der Jugendlichen auftauchen. Anschliessend folgen die Konzeptualisierung von Bricolage und die soziolinguistische Analyse des Beispiels.

\section{Exemplum: tuiback}

Das Beispiel tuiback (,Tabak') findet in folgendem Kontext statt: Andreas - ein Nidwaldner ${ }^{1}$ Gymnasiast - sitzt mit drei Freunden um Mitternacht zusammen. Sie vertreiben sich die Zeit mit dem Rauchen einer Wasserpfeife und mit heiteren Gesprächen über Alltägliches. In der vorangehenden Sequenz sprechen die Jugendlichen über das Projekt, für welches sie das vorliegende Gespräch aufzeichnen. Andreas erklärt, dass Studierende die Aufnahmen auswerten werden, worauf Nico mit der ironischen Frage-Antwort-Sequenz reagiert studierst $d u$ ? - ja immer. David wechselt auf Zeile 03 abrupt das Thema und versucht, mehr Tabak aufzutreiben für das Nachfüllen der Wasserpfeife. Die sprachliche Umsetzung dieser einfachen Aufgabe steht in der nachfolgenden Analyse im Zentrum.

\section{Beispiel: tuiback}

P37: 145 (00:05:25-00:05:45)

Personen: Nico (NIC), David (DAV), Marco (MAR), Timo (TIM)

Datum: 09.04.07

Transkript: Galliker (für Transkriptionskonventionen und Dialektverschriftung cf. Galliker (2014: 67-72)

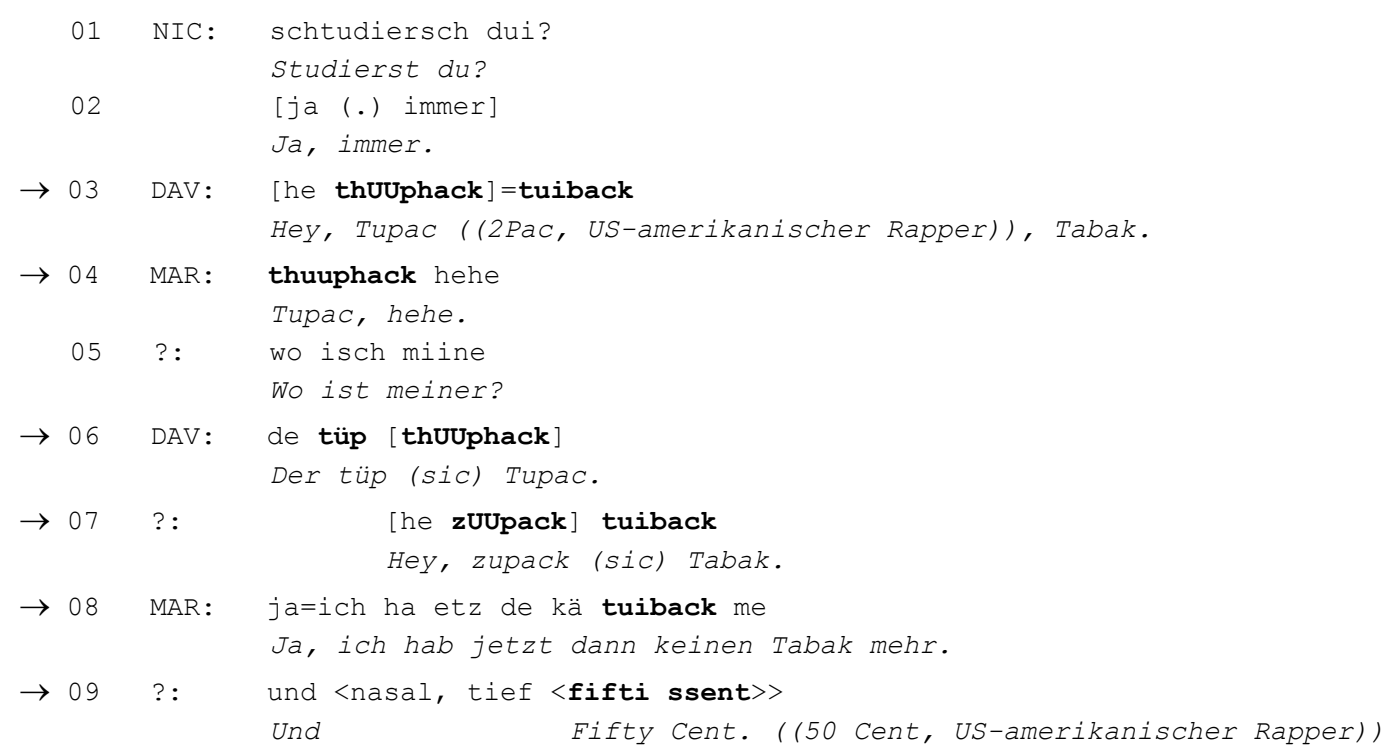

Abbildung 1: Beispiel für stilistische Variation zwischen lokalem Dialekt und „globalem Slang“6 (cf. Galliker 2014: 194)

Das Augenmerk bei der Betrachtung dieser Sequenz liegt primär auf der Variation des Begriffs ,Tabak'. Die erste Erwähnung findet der Begriff auf Zeile 03: David fragt seine Freun-

\footnotetext{
${ }^{1}$ Nidwalden ist ein kleiner Kanton in den zentralen Schweizer Alpen.
} 
de nach Tabak, indem er den dialektalen Begriff tuback zuerst mit aspiriertem $t$, einem gedehnten $u$ und einem aspirierten $p$ ausspricht, was eine stark markierte Aussprachevariante gegenüber der unmarkierten dialektalen Aussprache im Nidwaldner Dialekt darstellt. Diese Variation generiert den Eindruck, es handle sich dabei um den englischen Begriff Tupac (2Pac), den Namen eines US-amerikanischen Rappers. Das $a$ und das $k$ am Wortende werden jedoch in der schweizerdeutschen Aussprache belassen: als kurzes dunkles $a$, das zu $o$ neigt und als Affrikate $/ k x /$ (cf. auch Galliker 2014: 73-74). Die stilistische Vermischung von Englisch und schweizerdeutschem Dialekt bewirkt in diesem Fall, dass gleichzeitig nach Tabak gefragt wird sowie eine lautliche Anspielung auf den US-Hiphop und dessen Sprechstil umgesetzt wird.

David wiederholt den Begriff ,Tabak' direkt im Anschluss nochmals ( $Z$ 03), diesmal ohne Aspirationen, aber dafür mit einer ebenfalls markierten Aussprache des $u$ als Diphthong $u i$. In gewissen Nidwaldner Ortsdialekten wird das lange $u$ zu $u i$ diphthongiert (wie z.B. in huus > huis ,Haus', cf. Galliker 2014: 116). Dass hier nun jedoch das kurze $u$ in tuback zu tuiback diphthongiert wird, ist eine Art Übergeneralisierung der Jugendlichen und somit eine Anspielung an die Lautung des lokalen Ortsdialekts. Die ortsdialektale Variante ist in diesem Kontext eine stark markierte Variante, die von anderen Schülern und Schülerinnen des Gymnasiums in ihrer Binnenkommunikation kaum auf diese Weise verwendet würde.

Marco gefällt Davids erste Aussprachevariante (Englisch/Dialekt) offensichtlich und er wiederholt sie auf die gleiche Weise begleitet von einem honorierenden Lachen (Z 04). Nach einer Zwischenfrage greift David selber die erste Variante nochmals auf, um erneut nach Tabak zu fragen (Z 06).

Einer der Sprecher (der nicht identifiziert werden kann), bildet ad hoc dazu das Verb zuuphack (Z 07) und verbindet es mit dem sich darauf reimenden thuuphack. zuuphack ist eine morphologische Vermischung des hochdeutschen Verbzusatzes $z u$ und des schweizerdeutschen phackè. zuuphack wird also mit hochdeutschem Verbzusatz zuu (chdt. zuè) ausgesprochen und dieser nicht vom Verb abgespalten, was eine Normabweichung von der regulären dialektalen Syntax darstellt, die sonst hier verwendet würde. ${ }^{2}$ Also werden auch hier auf morphologischer resp. syntaktischer Ebene zwei Sprechstile resp. Sprachen (Schweizerdeutsch und Hochdeutsch) miteinander vermischt.

Marco wiederholt den Begriff nochmals in der übergeneralisierten ortsdialektalen Variante tuiback (Z 08) und bringt damit inhaltlich zum Ausdruck, dass ihm dieser nun endgültig ausgeht. Die Sequenz schliesst mit der betont nasal und englisch ausgesprochenen Aussage fifti ssent (50 Cent), wiederum dem Namen eines US-amerikanischen Rappers. Die englische, tiefe und nasale Aussprache sowie die Wiederaufnahme des Rapper-Themas verdeutlichen, dass auch dieser Jugendliche in der markierten Aussprache des Begriffs thuuphack auf Zeile 01 und Zeile 04 eine Anspielung auf den Rapper Tupac erkannt hat. Die inhaltliche Bedeutung dieses Turns ist in diesem Kontext sekundär, es geht lediglich um die Wiederaufnahme des englischen Hiphop-Stils. fifti ssent bedeutet hier etwa: ,genau! thuuphack tönt wie 2Pac und der kommt häufig mit 50 Cent vor".

\footnotetext{
2 Zuephacke gibt es auf Schweizerdeutsch auch, es kann allerdings nicht transitiv verwendet werden und zuu wird zuè ausgeprochen: pack zuè, aber nicht *pack de tuback zuè.
}

ISSN 1615-3014 
Im Anschluss an diese Sequenz wechseln die Jugendlichen das Thema und beginnen Witze aus deutschen Talkshows zu zitieren.

Das folgende vereinfachende Strukturschema soll nochmals die im Beispiel ablaufenden Variationsprozesse verdeutlichen:

\begin{tabular}{|c|c|c|c|c|}
\hline & Struktur & Bedeutung & Prozess & Sprecher \\
\hline 0 & $\begin{array}{c}\text { (tuback) } \\
\downarrow\end{array}$ & $($, Tabak') & $\begin{array}{c}\text { Vorgabe, nicht explizit erwähnt } \\
\downarrow\end{array}$ & - \\
\hline 1 & $\begin{array}{l}\text { thuuphack } \\
\downarrow\end{array}$ & ,Tabak' + ,Tupac' & $\begin{array}{c}\text { Transformation von } 0 \text { (Stil + Inhalt }) \\
\downarrow\end{array}$ & A \\
\hline 2 & $\begin{array}{l}\text { tuiback } \\
\quad \downarrow\end{array}$ & ,Tabak' & $\begin{array}{c}\text { Transformation von } 1(\text { Stil + Inhalt }) \\
\downarrow\end{array}$ & A \\
\hline 3 & $\begin{array}{c}\text { thuuphack } \\
\downarrow\end{array}$ & ,Tabak’ + ,Tupac’ & $\begin{array}{c}\text { Übernahme von } 1 / \text { Kontrast zu } 2 \text { (Stil + Inhalt) } \\
\downarrow\end{array}$ & B \\
\hline 4 & $\begin{array}{l}\text { thuuphack } \\
\qquad\end{array}$ & $"$ & $\begin{array}{c}\text { Übernahme von } 3 \\
\downarrow\end{array}$ & A \\
\hline 5 & $\begin{array}{c}\text { zuuphack tuiback } \\
\downarrow\end{array}$ & $\begin{array}{l}\text {,zupacken', Ta- } \\
\text { bak' }\end{array}$ & $\begin{array}{c}\text { Transformation von } 4 \text { (Form }+ \text { Inhalt), } \\
\text { Übernahme von } 2 / \text { Kontrast zu } 4 \\
\text { (Stil und Inhalt) } \\
\downarrow\end{array}$ & $\mathrm{C}$ \\
\hline 6 & $\begin{array}{l}\text { tuiback } \\
\quad \downarrow\end{array}$ & ,Tabak' & $\begin{array}{c}\text { Übernahme Teil von } 5 \\
\downarrow\end{array}$ & B \\
\hline 7 & fifti ssent & ,50 Cent' & Übernahme von 4 (Stil)/Kontrast zu 6 (Stil) & $?$ \\
\hline
\end{tabular}

Tabelle 1: Strukturschema Beispiel 1 (cf. Galliker 2014: 197)

Strukturell fällt auf, dass in diesem Gespräch der unmarkierte Dialektbegriff tuback (Schritt 0, im Gespräch nicht erwähnt) in eine englisch/schweizerische Variante transformiert wird und dadurch inhaltlich polysem wird (er bedeutet gleichzeitig ,Tabak' und ,Tupac', Schritte, 1, 3 und 4). Weiter wird der Begriff in der gleichen Sequenz auch in eine übergeneralisierte ortsdialektale Variante transformiert, die mit der englisch/schweizerdeutschen Variante kontrastiert (tuiback, Schritte 2 und 6). In einem weiteren Prozess bilden die Jugendlichen einen sich reimenden Neologismus zur englisch/schweizerdeutschen Variante (zuuphack tuiback, Schritt 5) und im letzten Schritt wird durch die Aussprache eines englischen Namens eine Anspielung auf den ganzen kommunikativen Stil der angloamerikanischen Hiphop-Szene vorgenommen (fifty sènt, Schritt 6).

Die Jugendlichen bedienen sich also bei einer simplen Aufgabe wie der Suche nach Tabak verschiedener sprachlicher Ressourcen und kombinieren, parallelisieren und kontrastieren diese anhand eigener Regeln. Die Regelhaftigkeit und die Funktionen dieser Variation sind dabei für Aussenstehende schwer erkenn- und interpretierbar ohne fundierte Kenntnis des Repertoires der Jugendlichen und ihrer sozialen und kulturellen Bezüge zur Orts- und Gesamtgesellschaft. Etwas offensichtlicher ist, dass die Jugendlichen Freude an diesem lautlichen Spiel empfinden (cf. Lachen Z 04) und sich gemeinsam daran beteiligen (cf. die vielen Wiederholungen des Begriffs).

Im Folgenden wird nun das Beispiel in den erweiterten Forschungs- resp. Untersuchungskontext eingebettet. Auf diese Weise sollen die darin enthaltenen Bezüge sowie die musterhafte 
Umsetzung der sprachlichen Verfahren in den Gesprächen dieser Jugendlichen verdeutlicht werden.

\section{Theoretischer Rahmen: Ethnographie der Kommunikation}

Wie das Beispiel zeigt, benötigt eine präzise Analyse und Interpretation solcher Variationsprozesse und ihrer Funktionen fundiertes Wissen über das Variationsspektrum, in welchem sich die Mitglieder einer Sprechgemeinschaft bewegen. Es braucht Wissen über Regeln und Normen, die ihren Sprachgebrauch bestimmen, und es braucht Wissen über ihr erweitertes soziales und kulturelles Umfeld, um die Bezüge und Funktionen ihres sprachlichen Verhaltens verstehen zu können. Der hier verwendete Ansatz untersucht sprachliche Handlungen in konkreten Kommunikationssituationen und betrachtet sie als soziale und kulturelle Handlungen in einem erweiterten gesellschaftlichen Kontext: Die Ethnographie der Kommunikation nach Hymes $(1974,1979)$ und Gumperz (u. a. 1982, 1994). Diese geht von einem Kompetenzansatz aus und versteht sprachliches Handeln als Wissen und anwenden können von sprachlichen Normen und Regeln, die es braucht, um erfolgreich am Leben einer Gesellschaft partizipieren zu können. Sie konzentriert sich dabei insbesondere auf sprachliches Handeln als Mittel, um gesellschaftliche Aufgaben und Probleme zu lösen. Zudem versucht sie, Strukturen, Muster und deren spezifische Funktionen herauszuarbeiten, die sich in der Kommunikation einzelner sozialer Gruppen dafür herausbilden. Als zentrale Ressource für sprachliche Handlungen betrachtet die Ethnographie der Kommunikation die Variabilität der Sprache. Das heisst, Sprachbenutzende können durch die Wahl zwischen verschiedenen Alternativen des Ausdrucks, durch die Verwendung von Variabilität als sprachliche Ressource - z. B. durch die Wahl zwischen diatopischen und diaphasischen Varianten - ausdrücken, wer sie sind, welcher Gruppe von Menschen sie sich zuordnen, wie sie sich und andere oder eine bestimmte Situation einschätzen. Auf diese Weise schaffen sie soziale Wirklichkeit. Dies alles ereignet sich in der engen und auch erweiterten Sprechgemeinschaft oder wie Gumperz es nennt, in „sozialen Netzwerken“ (Gumperz 1994: 623). Ein soziales Netzwerk ist eine Gruppe von Menschen, in welcher sich, über den anhaltenden sozialen Kontakt zwischen den Gruppenmitgliedern, geteilte sprachliche Ressourcen, Stile, Ansichten und Werte herausbilden, welche die (linguistische) Grundlage für lokale Kontextualisierungen in der Interaktion bilden, aber auch Bezüge zu grösseren sozialen Kategorien und Identitäten schaffen (cf. Galliker 2014: 29). Im Falle der untersuchten Jugendlichen geschieht das so in ihrer Peergruppe, in welcher sie gemeinsame Werte und eine Gruppenidentität teilen und diese auch über die geteilten sprachlichen Praktiken - den eigenen Gruppenstil - manifestieren. Die sprachlichen Handlungen der Jugendlichen können jedoch nicht isoliert betrachtet werden, auch die Identität der Gruppe definiert sich immer erst in Abgrenzung zu anderen sozialen Netzwerken anderen Jugendgruppen oder auch der erweiterten Sprechgemeinschaft im Elternhaus, dem Dorf und der Region.

In Bezug auf das oben stehende Beispiel bedeutet der ethnographische Zugang, dass die verschiedenen sprachlichen Varianten, die hier vorkommen, alle als stilistische Merkmale aufgefasst werden, welche die Jugendlichen aus ihrem Repertoire auswählen können, unabhängig davon, ob sie aus linguistischer Perspektive einer Sprache, einem Dialekt oder einem Sprechstil zuzuordnen sind (cf. Galliker 2014: 24). Trotzdem gibt es so etwas wie eine unmarkierte sprachliche Lage, derer sich die Jugendlichen untereinander bedienen und in Bezug auf wel-

ISSN 1615-3014 
che verschiedene sprachliche Varianten als markiert erscheinen. Die unmarkierte Lage konzeptualisiert Kallmeyer (1995) als Referenzsystem für die Variationsprozesse. Er nennt sie „Normallage“. Kallmeyer versteht dabei Normallage als „diatopische Normallage“, d. h. als Einordnung der unmarkierten Sprechlage einer Sprechgemeinschaft im diatopischen Variationsspektrum. Die hier eingesetzte Konzeption der Normallage folgt einer Erweiterung dieser Auffassung nach Augenstein (1998: u. a. 109 und 121): Sie geht davon aus, dass die Normallage auch im diaphasischen Variationsspektrum verortet werden muss und ausserdem verschiedene Normallagen für verschiedene Kontexte existieren. Analog dazu wird hier von zwei Normallagen ausgegangen, welche in das oben beschriebene Variationsspektrum in Bezug auf diatopische sowie diaphasische Aspekte eingeordnet werden: Erstens wird die unmarkierte Sprechlage beschrieben, die die Jungengruppe mit anderen Jugendlichen am Gymnasium und im erweiterten sprachlichen Umfeld mit jungen Mitgliedern der Sprechgemeinschaft anwendet. Sie wird hier als „Normallage OJ“, also als Normallage mit Out-Group-Jugendlichen in Nidwalden bezeichnet. Zweitens wird die Normallage beschrieben, die die Jugendlichen üblicherweise in der In-Group, also in der Binnenkommunikation der Peergruppe verwenden. Sie wird dementsprechend „Normallage IJ“ genannt (cf. Galliker 2014: 119 und unten Kp. 5).

\section{Daten und Datenerhebung: emische und etische Perspektive}

Die hier vorgestellten Daten und Ergebnisse stammen aus der Dissertation Bricolage. Ein kommunikatives Genre im Sprachgebrauch Jugendlicher aus der Deutschschweiz (Galliker 2014). Sie wurden im Rahmen des Projektes „Jugendsprache(n) in der Deutschschweiz“ erhoben, das von 2006 bis 2008 vom Schweizerischen Nationalfonds (SNF) gefördert wurde und unter der Leitung von Prof. Erika Lang an der Zürcher Hochschule für Angewandte Wissenschaften (ZHAW) angesiedelt war. Die Datenerhebung für das Dissertationsprojekt wurde bis 2010 weitergeführt (cf. Galliker 2014: 49, 60-67).

Dem ethnographischen Ansatz entsprechend wurde der Feldzugang über verschiedene Instrumente der Datenerhebung wie Aufzeichnungen von Binnenkommunikation, Interviews, Fragebogenerhebungen und Feldbeobachtungen gestaltet. Es wurde Wert darauf gelegt, die unterschiedlichen Erhebungsinstrumente und das daraus entstandene Datenmaterial sowie diverse emische (Innenperspektiven) und etische Perspektiven (Aussenperspektiven, cf. Gumperz 1982: 15 nach Pike 1964) auf den Untersuchungsgegenstand miteinander zu triangulieren. Ziel dieses Vorgehens war es, zu möglichst aussagekräftigen und validen Interpretationen des Materials zu gelangen (cf. Galliker 2014: 43-67, 85-88). Im Rahmen der Dissertation wurde u. a. eine Gruppe junger Gymnasiasten über ein Jahr lang ethnographisch begleitet. Die Jugendlichen selber erhielten in dieser Zeit ein Aufnahmegerät zur Aufzeichnung ihrer Binnenkommunikation (ca. 18 Stunden), d. h. ihrer Kommunikation untereinander, ohne das Beisein von Erwachsenen und auch Forschenden. Gleichzeitig wurden mit den wichtigsten Mitgliedern der Gruppe mehrere Interviews und offene Gespräche geführt (ca. 8 Stunden), in welchen sie zu ihren sprachlichen Praktiken befragt wurden. Teilweise wurden ihnen auch Gesprächsausschnitte aus ihren eigenen Aufnahmen vorgespielt, wodurch ihre persönlichen Meinungen und Interpretationen der sprachlichen und sozialen Prozesse abgefragt werden konnten (emische Perspektive). Auch Fragen und Interpretationen der Forschenden (etische Perspektive) konnten geklärt und validiert werden. Die Innenperspektive der Gymnasiasten trug wesentlich zum Verständnis der Daten und auch zu ihrer Verortung im Meso- und Mak-

ISSN 1615-3014 
rokontext bei. Zur Vervollständigung wurden Interviews und Gespräche mit anderen Schülerinnen und Schülern aus den Klassen der Peers sowie mit deren Lehrpersonen geführt. Feldbeobachtungen (z. B. in öffentlichen Verkehrsmitteln und an kulturellen Anlässen) sowie ein Set an nicht-reaktiven Daten (z. B. eine von den Peers erstellte Homepage) ergänzen das Bild. Eine weitere teilweise emische sowie auch etische Perspektive auf den Untersuchungsgegenstand ergab sich daraus, dass die Forscherin selber im Kanton aufgewachsen ist, dasselbe Gymnasium besuchte und immer wieder für Familienbesuche und den Besuch kultureller Anlässe in der Gegend war. Aus diesem Grund ist sie mit der dialektalen Situation im Kanton vertraut. Zum Zeitpunkt der Datenerhebung wohnte und arbeitete sie jedoch schon mehrere Jahre ausserhalb des Kantons und ein Blick aus einer Aussenperspektive konnte so ebenfalls gewährleistet werden (cf. Galliker 2014: 58-59).

Für die Analyse der Daten wurden diese transkribiert und mit Methoden der ethnographischen Gesprächsanalyse (u. a. Deppermann 2000) und linguistischen Beschreibungsmethoden bearbeitet (cf. Galliker 2014: 72-75). Für die Konzeptualisierungsarbeit resp. das theoretische Codieren wurde die Analysesoftware Atlas.ti verwendet (ibd.)

\section{Ethnographie der Nidwaldner Peergruppe}

In der nachfolgenden knappen ethnographischen Beschreibung der Peergruppe wird v. a. auf jene Aspekte fokussiert, welche relevant sind für die Analyse und Interpretation des obenstehenden Beispiels.

Bei der Peergruppe handelt es sich um ein mehr oder weniger offenes, flexibles soziales Netzwerk bzw. eine Sprechgemeinschaft (Gumperz 1994: 623), die geprägt ist von einer Kerngruppe von 7 Gymnasiasten und ca. 15 weiteren männlichen, 16- bis 18-jährigen Jugendlichen. Diese besuchen gemeinsam das Kollegium St. Fidelis - die einzige Mittelschule im ländlichen Kanton Nidwalden in den Zentralschweizer Alpen. Dort gibt es immer noch ein aktives traditionelles Kultur- und Dorfleben sowie viele Brauchtümer (wie die Fastnacht, das Erntedankfest (Älplerchilbi), Einzug des hl. Nikolaus und sportliche Anlässe wie das Schwingen (lokale Art des Ringens) etc.). Der Ausländeranteil in der Bevölkerung und am Gymnasium ist im Vergleich zu anderen Regionen tief (10\% in Nidwalden, cf. Nidwaldner Kantonalbank 2007: 4, im Vergleich zu 23\% in Luzern oder 31\% in Zürich, cf. Lustat: 2011, cf. auch Galliker 2014: 90-100).

Die Jugendlichen verbringen einen Grossteil ihrer Schul- und Freizeit miteinander. Über diesen sozialen Kontakt in ihrem Netzwerk haben sie im Verlauf ihrer Freundschaft einen eigenen, für sie typischen sozialen kommunikativen Sprechstil herausgebildet. Darunter wird nach Gumperz resp. Kallmeyer und Keim (u. a. 2002: 37) ein Stil verstanden, der dazu dient, die soziale Positionierung der Sprechenden anzuzeigen und zu kontextualisieren und dadurch soziale Identität zu konstituieren (cf. Galliker 2014: 19-23, 159-175).

Um beschreiben zu können, inwiefern sich der Sprechstil der Peergruppe als einzigartiger Gruppenstil oder eben als kommunikativer sozialer Stil auszeichnet, braucht es ein Referenzsystem für die Variation. In der binnendeutschen Jugendsprachforschung wird seit jeher die Standardsprache als Referenzsystem verwendet, um aufzuzeigen, wie Jugendliche in ihrem Sprechverhalten davon abweichen. Dieser Prozess wird als „Substandardisierung“ bezeichnet (cf. u. a. Neuland 2001). In der Deutschschweiz ist dieses Vorgehen nicht umsetzbar, da die 
Jugendlichen ausschliesslich in formellen (schulischen) Kontexten hochdeutsche Standardsprache sprechen (cf. Galliker 2014: 104-119). In jeder anderen Situation ist die erste Wahl der (Nidwaldner) Dialekt, d. h. ihre Normallage ist die dialektale Variation (das gilt i. Ü. auch für erwachsene Sprechende). Diese Normallage im Dialekt ist also die unmarkierte Sprechlage, welche die Gruppe in ihrer Binnenkommunikation verwendet. Sie dient als Referenzsystem für die Beschreibung von Variationsphänomenen und zeichnet sich durch besondere Merkmale in Bezug auf die diaphasische sowie die diatopische Variation aus.

Auch wenn Jugendliche und Erwachsene gleichermassen Dialekt als sprachliche Normallage verwenden, ist die dialektale Normallage der GymnasialschülerInnen untereinander nicht identisch mit jener älterer Sprechender in den umliegenden Dörfern: Es hat sich gezeigt, dass viele Jugendliche in ihren Heimatdörfern mit lokalen Ortsdialekten aufwachsen. Sobald sie aber ans Gymnasium gehen und den ganzen Tag mit Peers aus verschiedenen Ortschaften und mit z. T. weniger starken Dialektausprägungen verbringen, legen sie die markantesten Dialektmerkmale $\mathrm{ab}$ und wechseln sozusagen in eine Koine, also in einen regionalen Dialekt. Diese dialektale Lage ist immer noch als Nidwaldner Dialekt erkennbar, es fehlen ihm jedoch die salientesten Merkmale (für eine detaillierte Beschreibung cf. Galliker 2014: 115-126). Zu beobachten ist zudem, dass alle Jugendlichen der Peergruppe sowie auch ihres erweiterten Umfeldes dazu tendieren, dialektale Lexeme mit standardnahen Lexemen in dialektaler Aussprache zu ersetzen (eine Liste findet sich in Galliker 2014: 125-126).

Der folgende Satz soll die zwei dialektalen Lagen auf lautlicher Ebene exemplarisch verdeutlichen: Im Nidwaldner Ortsdialekt z. B. aus Wolfenschiessen würde der Satz Mein Bruder hätte gerne tausend schöne kleine Mäuse wie folgt lauten:

1) meii briäder hätt gärä tuisig scheeni chleini meisli

In der Koine könnte der Satz z. B. so lauten:

2) mii brüäder hätt gärn tuusig schööni chliini müüsli

Die salienten Merkmale des Ortsdialektes gegenüber der Koine sind also die zerdehnte, diphthongartige Aussprache der Langvokale $i$ und $\ddot{u}$ in ei (/ei/, miis vs. meis, müüsli vs. meisli) und die Diphthongierungen des langen $u$ in $u i$ (/ui/, tuusig vs. tuisig) sowie die Entrundung von $\ddot{o}$ zu e (schööni vs. scheeni).

Auch die Mitglieder der hier im Fokus stehenden Peergruppe bewegen sich sprachlich zwischen diesen beiden Polen Ortsdialekt und Koine. Diejenigen, die zu Hause oder in der Grundschule einen lokalen Ortsdialekt erlernt haben, verwenden ihn dort mit ihren Eltern und Peers. Wenn sie jedoch ans Gymnasium gehen und sich mit anderen Jugendlichen ausserhalb ihrer Peergruppe unterhalten, schwächen sie die salienten Merkmale des Ortsdialektes ab. Sie unterscheiden sich aber deutlich in ihrem sprachlichen Verhalten, wenn sie innerhalb der Peergruppe kommunizieren: Dann orientieren sie sich im Unterschied zu den anderen Schülern und Schülerinnen stark am lokalen Ortsdialekt. So verwenden sie verschiedene aus ihrer Sicht urtümliche Lautvarianten, Lexeme, bestimmte prosodische Merkmale und teilweise auch stilisierte Varianten des Ortsdialektes. Besonders diese stilisierten Varianten des Ortsdialektes verbinden sie stark mit dem Konzept des „Urchigen“, wie sie es selber im Dialekt nennen. Das „Urchige“ - gemeint ist eine urtümliche Sprechweise wie auch das urtümliche, traditionelle Brauchtum, verknüpft mit einer ländlichen, bäuerlichen Lebensweise - ist bei

ISSN 1615-3014 
den Jugendlichen in der Peergruppe hoch angesehen. Bedenkt man, dass von ihnen selber keiner aus einer Bauernfamilie stammt und ihre diesbezügliche soziale Distanz über ihre schulische Ausbildung an der Mittelschule recht gross ist, ist diese Orientierung am traditionellen Kulturgut und Brauchtum etwas Besonderes (cf. Galliker 2014: 143-149) und stellt ein Alleinstellungsmerkmal gegenüber den anderen Schülern und Schülerinnen des Gymnasiums dar.

Die folgende schematische Zusammenfassung verdeutlicht die Bezüge der Peergruppe über ihre diatopische Variation zu anderen sozialen Gruppen auf der Mikro-, Meso- und Makroebene. Die Übersicht in Tabelle 2 zeigt, wie sich die Jugendlichen durch ihre diatopische Variation auf der Mesoebene und auf der Makroebene positionieren. Es wird deutlich, dass sie sich durch die Ansiedlung ihrer Normallage in der Peergruppenkommunikation im Bereich des Ortsdialektes nicht primär von den Erwachsenen unterscheiden, sondern von den anderen Schülern und Schülerinnen des Kollegiums. Der urtümliche Ortsdialekt dient also an der Schule zur Markierung von Gruppenzugehörigkeit zur Peergruppe. Dennoch ist die Art ihrer Verwendung des Ortsdialektes auch klar unterscheidbar von jener der Erwachsenen insbesondere über Stilisierungen und die Verwendung von Bricolagen (s. u.) mit dem Ortsdialekt.

Ortsdialekt

Regionaler NW-

Standard Dialekt

\begin{tabular}{ll|ll}
$\begin{array}{l}\text { Makroebe- } \\
\text { ne }\end{array}$ & $\begin{array}{l}\text { Erwachsene, } \\
\text { einheimische } \\
\text { NidwaldnerIn- } \\
\text { nen }\end{array}$ & $\begin{array}{l}\text { Mit nicht-autochthonen } \\
\text { DeutschschweizerInnen } \\
\text { z. B. beim Einkaufen in Lu- } \\
\text { Normallage }\end{array}$ & $\begin{array}{l}\text { Mit Leuten, die } \\
\text { kein Schweizer- } \\
\text { deutsch verste- } \\
\text { hen, }\end{array}$ \\
& & $\begin{array}{l}\text { im schulischen } \\
\text { und tlw. im be- } \\
\text { ruflichen Kon- } \\
\text { text }\end{array}$ \\
& &
\end{tabular}

$\begin{array}{clll}\text { Mesoebene } & \text { GymnasiastIn- } \\ \text { nen } & \text { Tlw. zu } & & \text { Mit Leuten, die } \\ & \text { Hause, } & \text { kein Schweizer- } \\ & \text { mit Leu- } & & \text { deutsch verste- } \\ & \text { ten im } & \text { hen, } \\ & \text { Dorf } & \text { Normallage } & \text { im Schulunter- } \\ & & \text { richt } \\ & & \\ & & \end{array}$

Mikroebe- Peergruppe
ne

Mikroebe- Peergruppe ne
Mit Leuten, die kein Schweizerdeutsch verstehen, im schulischen ruflichen Kontext
Mit Leuten, die kein Schweizerisch versteim Schulunter-

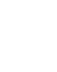


hen,

im Schulunter-

richt

Tabelle 2: Übersicht der Bezüge zu Mikro-, Meso- und Makroebene der Peergruppe über diatopische Variation (cf. Galliker 2014: 180)

Nebst diesen Formen dialektaler Variation verfügen die Jugendlichen auch über ein breites Spektrum an weiteren diatopischen, sprachlichen Ressourcen, welche hier nicht im Detail beschrieben werden können. So verwenden sie beispielsweise binnendeutsches Hochdeutsch, Englisch, Französisch und Spanisch als Versatzstücke in ihren Gesprächen sowie verschiedene stilistische Ausprägungen innerhalb dieser Varietäten, wie etwa ethnolektale Sprechweisen des Deutschen (in der Schweiz umgangsspr. "Balkanslang") oder des Englischen (z. B. Hiphop Slang), (cf. Galliker 2014: u. a. 140-149).

Unterschiedliche stilistische Lagen verwenden die Jugendlichen auch innerhalb des Nidwaldner Dialektes. Auf diaphasischer Ebene können analog zur diatopischen Ebene zwei Normallagen unterschieden werden: Diejenige, die sie mit den Out-Group-Jugendlichen am Gymnasium verwenden, und jene in der Peergruppe. Wenn die Jugendlichen mit ihren Schulkameraden und -kameradinnen sprechen, verwenden sie viele sprachliche Merkmale, welche bereits mehrfach für binnendeutsche oder anderssprachige Jugendstile, besonders auch in urbanen und mehrsprachigen Kontexten als typisch charakterisiert wurden (detailliert z. B. in Henne 1986, Androutsopoulos 1997, Neuland 2008, Jørgensen et al. 2011, in Bezug auf Nidwalden Galliker 2014: 126-140). Dies ist beispielsweise die Verwendung einer stark gruppenspezifischen Lexik u. a. auch angereichert mit Fachbegriffen aus unterschiedlichen Jugendkulturen, vermehrter Gebrauch von Anglizismen und Verstärkungspartikeln, häufige Verwendung jugendtypischer Interjektionen und Phraseologismen, z. T. derbe, hyperbolische, ironische und stilisierende Sprechweisen. Partiell überschneiden sich die verwendeten sprachlichen Varianten auch mit denjenigen z. B. deutschsprachiger Jugendlicher aus Deutschland wie die Ansprache von Peers mit alter und dessen Verwendung als Gesprächspartikel. Andererseits gibt es auch spezifische stilistische Merkmale, welche regional beschränkt sind wie die Verstärkungspartikel huèrè (=,sehr'/'extrem' cf. Galliker 2014: 126-127).

Sprechen nun die Peergruppenmitglieder nur untereinander, verwenden sie einerseits die weiter verbreiteten Merkmale, andererseits jedoch auch solche mit stark gruppenspezifischer Ausprägung. So integrieren sie beispielsweise Laute in ihren Sprechstil, die in ihrem Dialekt nicht standardmässig vorkommen (z. B. verwenden sie den im Albanischen gebräuchlichen retroflexen Approximanten [.] anstelle des apikalen Vibranten $/ \mathrm{r} /$ in Namen der Peers wie ma.co, ibd. 150). Oder sie verwenden einzelne ortsdialektale Laute auf gruppenspezifische Weise (cf. tuiback in diesem Artikel oder auch z. B. in meittwuch (=Mittwoch), ibd. 188). Auch auf morphologischer Ebene geht die Gruppe eigene Wege, indem z. B. Morpheme verschiedener Varietäten miteinander kombiniert werden (cf. zuuphack in diesem Beispiel oder piiken (= engl. peak und hdt. -en für ,hoch ausschlagen'), ibd. 224). Lexikalisch verwenden die Jugendlichen gerne Dialekt- oder andere Ausdrücke und desemantisieren diese (fifty sent in diesem Beispiel, wüete (hdt. wüten) für ,Party machen', riämä (hdt. Riemen) u. a. für ,Pe- 
nis', ibd. 259). Sie integrieren in sehr freier Weise verschiedene Sprechstile und Sprachen in ihren eigenen Stil (z. B. den "urchigen" Stil, ibd. 143ff.) und haben eine Vorliebe für ironische und hyperbolische Sprechweisen. Auffällig bei all diesen sprachlichen Prozessen ist, dass die Jugendlichen der Peergruppe diese gemeinsam in musterhaft ablaufenden BricolageProzessen generieren. Bricolagen auf allen Ebenen des Sprachsystems können deshalb als stilistisches Kernelement des Gruppenstils betrachtet werden (für die Konzeptualisierung von Bricolage cf. Kp. 6).

In der untenstehenden Übersicht werden die sozialen Bezüge verdeutlicht, welche über die diaphasische Variation der Jugendlichen hergestellt werden können. Der gruppenspezifische Stil erweist sich dabei als Stil, der seine soziale Bedeutung vor allem auf der Mikro- und Mesoebene entfaltet. Die Jugendlichen positionieren sich so einerseits durch sprachliche Vergemeinschaftung und Sprachprofilierung innerhalb ihrer Peergruppe, andererseits grenzen sie sich indirekt von den anderen Jugendlichen in ihrem Umfeld ab. Das geschieht, indem sie mit der Peergruppe den Gruppen-Stil verwenden und mit aussenstehenden Jugendlichen die in diesem Kontext unmarkierte „Nidwaldner Jugendsprache“ (Normallage OJ = Out-Group Jugendliche).

\begin{tabular}{|c|c|c|c|c|}
\hline & & Gruppen-Stil & $\begin{array}{c}\text { „Nidwaldner Jugend- } \\
\text { sprache“ }\end{array}$ & $\begin{array}{c}\text { „Nidwaldner } \\
\text { Umgangssprache“ }\end{array}$ \\
\hline Makroebene & $\begin{array}{l}\text { Erwachsene, } \\
\text { einheimische } \\
\text { NidwaldnerInnen }\end{array}$ & --- & $\begin{array}{l}\text { selten als Abgren- } \\
\text { zung und Markie- } \\
\text { rung von Jugend- } \\
\text { lichkeit }\end{array}$ & Normallage \\
\hline
\end{tabular}

Mesoebene GymnasiastInnen Tlw. als Fremdstilisierung der Peergruppe
Mit Erwachsenen und Älteren

Normallage

Mikroebene Peergruppe
Normallage
Mit Gymnasias-

tInnen, mit Jugendlichen der Out-Group
Mit Erwachsenen und Älteren

Tabelle 3: Übersicht über Bezüge zur Mikro-, Meso- und Makroebene der Peergruppe über diaphasische Variation (cf. Galliker 2014: 179) 


\section{Bricolage}

Wie bereits erwähnt, können sehr viele der für den Gruppenstil spezifischen sprachlichen Merkmale - wie sie auch im Beispiel vorkommen - über das Konzept der Bricolage erfasst und beschrieben werden. Das Konzept der Bricolage geht auf Lévi-Strauss (1963) resp. Clarke et al. $(1975,1979)$ zurück und lässt sich auf folgendes einfaches Grundprinzip reduzieren: Bestehende soziale oder kulturelle Ressourcen werden von Gesellschaftsmitgliedern aufgegriffen und so transformiert, dass dadurch etwas Neues und Eigenes entsteht. Im Sinne eines sensitivierenden Konzeptes (Denzin 1989: 14) wurde dieses Konzept auf die sprachlichen Daten der Dissertation angewandt, welche diesem Artikel zugrunde liegen (cf. Galliker 2014). Es wurde ausgehend von detaillierten linguistischen Analysen des Korpus ausgearbeitet und präzisiert. Die dabei entstandene Konzeptualisierung soll hier zusammenfassend präsentiert und anhand des obenstehenden Beispiels erläutert werden.

\subsection{Bricolagen im Exemplum}

Werden die Darstellungen zu den sprachlichen Ressourcen resp. des Variationsraumes auf das eingangs präsentierte Beispiel bezogen, lässt sich Folgendes resümieren: Die Jugendlichen starten in ihrer sprachlichen Normallage der In-Group, d. h. dem Nidwaldner Ortsdialekt.

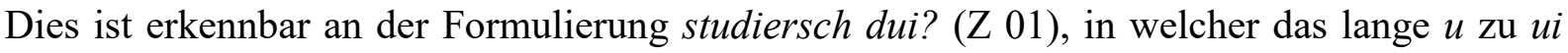
diphthongiert ist. In den nachfolgenden stilistischen Basteleien wird abwechslungsweise auf lautlicher $(Z$ 03, 04, 06, 08) und auf morphologischer ( $Z$ 07) sowie auf stilistischer Ebene ( $Z$ $09)$,gebastelt", indem das englische 50 Cent auch prosodisch durch Tiefe und Nasalität gerahmt wird.

All diese sprachlichen Prozesse können als Bricolagen aufgefasst werden, da bestehende sprachliche Ressourcen, welche mit kulturellen resp. sozialen Bedeutungen verknüpft sind, von den Jugendlichen zu einem eigenen Sprechstil zusammengefügt werden. Die Bricolagen können entsprechend der sprachlichen Ebene resp. Einheit der vorkommenden Ressourcen unterschieden werden z. B. in lautliche, morphologische und stilistische Bricolagen (s. u.). Mit der lautlichen Bricolage - der Übergeneralisierung von ui in tuiback - wird hier Bezug genommen auf den urtümlichen Nidwaldner Dialekt und das lokale Brauchtum ${ }^{3}$, mit der morphologischen Bricolage auf deutsche Standardsprache und ihre Normen und mit der stilistischen Bricolage schliesslich auf die weit verbreitete Jugendkultur des angloamerikanischen Hiphops.

\footnotetext{
${ }^{3}$ Diese Darstellung des Bezuges zum Brauchtum über den Ortsdialekt scheint an dieser Stelle evt. etwas verkürzt. Die Jugendlichen verwenden den lokalen Ortsdialekt jedoch häufig miteinander in Form eines kommunikativen sozialen Stils (Kallmeyer/Keim (2003: 38) zur Fremddarstellung. Sie beziehen sich damit auf aus ihrer Sicht „urchige“, also urtümliche Mitglieder der Gesellschaft wie Schwinger (lokale Form von Ringern), Müllmänner oder Bauern. Für die Jugendlichen stellen diese stereotype Vorbilder dar, welchen sie eine direkte und schroffe Art des Sprechens zuschreiben und einen engen Bezug zu lokalen Traditionen über den Schwingsport und das Älplertum (Brauchtum der Bauern in den Alpregionen). Das lokale Brauchtum hat für die Jugendlichen einen hohen Stellenwert und über die Stilisierungen des lokalen Ortsdialektes stellen sie in ihren Gesprächen einen Bezug dazu her. Eine fundierte Beschreibung des Brauchtums und des entsprechenden Stils findet sich in Galliker 2014: 89-96 und 143-149). Der Stil ist so stark verfestigt im Sprachgebrauch der Jugendlichen, dass sie bereits mit dem Einflechten eines einzelnen typischen Lautes wie dem $u i$ auf den Stil und den damit verknüpften sozialen Handlungen wie dem Brauchtum Bezug nehmen können.
}

ISSN 1615-3014 


\begin{tabular}{|c|c|c|c|c|}
\hline 01 & NIC : & schtudiersch dui? & Normallage IJ: Nidwaldner Ortsdialekt & \\
\hline 02 & & {$[j a$ (.) immer $]$} & & \\
\hline 03 & DAV : & [he thuUphack] =tuiback & $2 \times$ Lautliche Bricolagen & $\sum$ \\
\hline 04 & MAR: & thuuphack hehe & Lautliche Bricolage & . \\
\hline 05 & ?: & wo isch miine & & $\overrightarrow{0}$ \\
\hline 06 & DAV : & de tüp [thUUphack] & Lautliche Bricolage & : \\
\hline 07 & ?: & [he zUUpack] tuiback & Morphologische Bricolage & $\stackrel{0}{2}$ \\
\hline 08 & MAR: & ja=ich ha etz de kä tuiback me & Lautliche Bricolage & \\
\hline 09 & ?: & und <nasal, tief <fifti ssent $>>$ & Stilistische Bricolage & \\
\hline
\end{tabular}

Tabelle 4: Übersicht über Bricolagen im Exemplum

\subsection{Bricolage als kommunikatives Genre}

Wie im Beispiel ersichtlich, lassen sich also hintereinander verschiedene Bricolage-Prozesse auf unterschiedlichen sprachlichen Ebenen festmachen, was zu einer Art ,stilistischem Schillern“ im Gespräch führt. Hintereinander werden in einem sehr schnellen und routinierten Ablauf von mehreren Gesprächsteilnehmern die verschiedensten Stile und Stilelemente beigesteuert, parallelisiert und miteinander kontrastiert. Gelungene Beiträge werden entweder wiederaufgenommen und honoriert (Lachen auf Z 04) oder weiterentwickelt ( $\mathrm{Z} 07$ resp. Z 09). Dabei sind bei der hier untersuchten Gruppe sprachliche Kreativität und Schnelligkeit sehr angesehen. Es gilt sozusagen, immer eine noch gelungenere Variante draufzusetzen und die Variationsprozesse gekonnt weiterzuspinnen. Funktional geht es eigentlich darum, Tabak aufzutreiben: Der erste Junge, David, fordert die anderen zur Mithilfe auf (direktive Funktion). Diese Funktion scheint aber während der ganzen Sequenz immer wieder in den Hintergrund zu rücken. Vielmehr versuchen sich die Jugendlichen sprachlich selber als Gruppenmitglied darzustellen (expressive Funktion) und sich zu vergemeinschaften (phatische Funktion), indem sie sich rege und kreativ am Bricolage-Prozess beteiligen. Auch die poetische Funktion, das sprachliche Spiel um seiner selbst willen, ist für die jungen Männer hierbei von Bedeutung (für eine fortgeführte Interpretation s. u., für die sprachlichen Funktionen cf. Hymes 1979: 64-60 resp. Galliker 2014: 16-19).

Das hier skizzierte kommunikative Verfahren (Gumperz 1994: 623) taucht im untersuchten Korpus der Peergruppe in musterhafter Weise und sehr häufig auf. Es ist geradezu ein Kernelement ihres Gruppenstils. In der Binnenkommunikation der Gruppe zeigen sich diese stilistischen Basteleien jedoch meistens nicht als isolierte kommunikative Verfahren wie z. B. in einer einzelnen lautlichen Bricolage, sondern wie hier als Kombination mehrerer Verfahren also als Verfahrensmuster - auf verschiedenen Ebenen des Sprachsystems. So dehnt sich die sprachliche Handlung oft auf die Mesoebene des Gespräches aus. Die Jugendlichen halten sich dabei an spezifische Normen und ein ganzes Set an prägenden Faktoren zeichnet die Sequenzen aus. Für die untersuchte Peergruppenkommunikation führte dies zu einer erweiterten Konzeptualisierung von Bricolage als komplexes kommunikatives ${ }^{4}$ Genre (nach Hymes 1974: 61, cf. Galliker 2014: 15). Hymes spricht von Genres, wenn Sprechereignisse relativ stark ritualisiert sind in Bezug auf die darin vorkommenden Sprechhandlungen (resp. kommunika-

\footnotetext{
${ }^{4}$ Kommunikativ deshalb, um es von anderen Typen des Genres abzugrenzen wie etwa mediale Genres (cf. Galliker 2014: 371).
} 
tiven Verfahren) und deren Abläufe sowie in Bezug auf Themen, Funktionen und Sprechende. Die folgenden neun Faktoren haben sich für die Bricolagen in den untersuchten Peergruppengesprächen als besonders charakteristisch herausgestellt und führten dementsprechend zu einer Konzeptualisierung einzelner Bricolagen als Genre (cf. Galliker 2014: 369-371):

a) Sprechende: Die Bricolagen sind interaktive Gruppenprozesse, die meistens gemeinsam von mehreren Sprechenden konstruiert werden.

b) Sequenzialität: Die Bricolagen enthalten folgende strukturelle Teile: Vorgabe Übernahme - Transformation. Typisch in dieser Abfolge ist die beliebig häufige Repetition meistens der beiden letzten Schritte. Zentrales Element ist der Transformationsprozess (bzw. die Verfremdung), um etwas Neues und Eigenes zu generieren. Die Bricolagen weisen also eine bestimmte Sequenzialität auf, jedoch ohne strikt definierte Abläufe. Zudem sind meistens ein klarer Anfang sowie ein Ende der Sequenz erkennbar. Die Bricolagen haben eine stark dialogische Struktur und sind häufig geprägt von der rhetorischen Figur der Steigerung.

c) Sprechhandlungen: Die zentralen Sprechhandlungen (resp. kommunikativen Verfahren) innerhalb der Bricolagen sind das Transformieren und Parallelisieren. "Transformieren bedeutet, dass bestehende Formen verändert werden, Parallelisieren bedeutet, dass z. B. Laute aus verschiedenen Varietäten oder ganze Stile durch die gleichzeitige Verwendung parallel zueinander eingesetzt werden. Diese beiden Sprechhandlungen zeichnen sich durch das Durchbrechen von Erwartbarem und das Verfremden von Elementen aller Ebenen des Ausdruckssystems aus und verfolgen dadurch das stilistische Grundprinzip des Kontrastierens. Die Verfremdungen spielen sich häufig auf struktureller Ebene ab, lassen sich aber ebenso auf konversationeller oder propositionaler Ebene wie auch gleichzeitig auf allen drei Ebenen erzeugen.

Häufig enthalten die Bricolagen auch zusätzliche charakteristische Sprechhandlungen wie Unterstellungen, Beleidigungen, Neckereien, Frage-Antwort-Sequenzen und chorales Sprechen (cf. Kp. 7.3).

d) Ressourcen: Für die Bricolagen können alle sprachlichen resp. kulturellen Ressourcen eingesetzt werden. Die Grösse der verwendeten Einheiten ist variabel und aus unterschiedlichen Ebenen des Ausdruckssystems: Laute, Morpheme, Lexeme, Phraseologismen, Dialekte, Stile, Sprachen, Texte, Genres, Ideen (Kleidern, Gegenständen, Bildern...) finden dabei Verwendung. Es wird auch mit den Mustern und Normen der entsprechenden Ressourcen gespielt und diese werden aufgegriffen, verändert und durchbrochen. Die Wahl der Ressourcen und ihre Bewertung sind stark abhängig vom betrachteten Netzwerk und der umgebenden Gesellschaft.

e) Funktionen: Bricolagen können, bedingt z. B. durch soziale Netzwerke und konkrete Verwendungssituationen, unterschiedliche Funktionen aufweisen. Im Korpus erwiesen sich die soziale (insbesondere auch in Form von exzessiven Selbstdarstellungen) und die poetische Funktion (Ästhetisierung) als zentral (s. u.) und die Jugendlichen setzten sie stark zur Vergemeinschaftung in der Gruppe ein. Grundsätzlich sind es also sym- 
metrische Prozesse, ${ }^{5}$ in welchen die Gesprächspartner einander gleichgestellt sind und sich über die gleichartige Verwendung und Bewertung von sprachlichen Ressourcen gegenseitig bestätigen.

Bricolagen können dementsprechend von den Sprechenden auf ihr Gelingen und ihre Qualität hin beurteilt werden.

f) Interaktionsmodalität: Die Bricolagen kontextualisieren meistens die Interaktionsmodalität Spass.

g) Interaktionsdynamik: Die Bricolagen sind häufig geprägt von Schnelligkeit, d. h. von schnellen Sprecherwechseln, schnellen Anschlüssen und Überlappungen, die Ausdruck der Routine im Umgang mit den Strukturen und Gesprächsabläufen und somit auch der Vertrautheit der Sprechenden miteinander sind.

h) Sprechsituation: Die Bricolagen treten vorwiegend in relativ unfokussierten Sprechsituationen unter vertrauten Personen auf. Häufig sind die Situationen geprägt von zeitlichem Überfluss ohne konkrete Aufgaben und teilweise auch durch Langeweile.

i) Thema: Die unterschiedlichsten Themen können im Zentrum einer Bricolage stehen. Sie sind abhängig von den Präferenzen des Netzwerks und reflektieren diese. Oft entfalten sie sich aus einer aktuellen Begebenheit und einem konkreten Kontext heraus.

Die hier für das Genre Bricolage beschriebenen prägenden Faktoren lassen sich alle auch im oben betrachteten Beispiel ausmachen: Mehrere Sprechende sind am Prozess beteiligt und nehmen ein Thema ad hoc aus der aktuellen Situation auf (Suche nach Tabak). Die Situation ist geprägt von zeitlichem Überfluss und Langeweile (Sprechsituation „Rumhängen“). Die Jugendlichen transformieren dabei bestehende sprachliche resp. kulturelle Ressourcen, indem sie verschiedene Varianten von Lauten, Morphemen und Stilen miteinander kombinieren, parallelisieren und auch kontrastieren. In der Konversation ist ein klarer Anfang dieses Prozesses ersichtlich, die Sequenz ist geprägt von Schnelligkeit und Steigerung (inhaltlich, zeitlich, konversationell) und wird auch klar wieder abgeschlossen mit dem Beitrag in der englischen Stilisierung. Die Bedeutung der Ressourcen sowie auch die enthaltenen „Normverstösse“ sind stark netzwerkspezifisch. Sie haben demzufolge eine soziale Funktion der Selbstdarstellung. Jugendliche, die diese Prozesse besonders gut beherrschen, also routiniert, schnell und kreativ sind, geniessen in der Gruppe besonderes Ansehen. Gleichzeitig entsteht durch das gemeinsame sprachliche Basteln auch ein Gefühl von Gruppenzugehörigkeit. Die Jugendlichen haben Spass am poetischen Spiel und drücken dadurch eine gewisse Nonchalance aus.

\subsection{Typisierung von Bricolagen}

Obwohl sich in den Gesprächen der hier untersuchten Jugendlichen Bricolagen sehr häufig in dieser erweiterten Form als Genre zeigen, ist diese nicht die einzige vorkommende Form. Es hat sich als hilfreich erwiesen, Bricolagen je nach verwendeten Ressourcen, nach Komplexitätsgrad und nach Verfestigungsgrad zu typisieren (cf. Galliker 2014: 377-384):

\footnotetext{
${ }^{5}$ Im Gegensatz etwa zu anderen jugendtypischen Genres wie etwa dem Dissen und Frotzeln (cf. Kp. 7.3).
} 


\subsubsection{Typisierung von Bricolagen nach Ressourcen und sprachlichen Einheiten: von lautlich $\mathrm{zu}$ intertextuell und ideell}

Die Bricolagen können nach der sprachlichen Einheit respektive Ressource unterschieden werden, welche in ihnen übernommen, transformiert und zu etwas Neuem gestaltet wird. Drei dieser Typen wurden oben bereits anhand des Beispiels aufgezeigt. Insgesamt lassen sich die folgenden sechs Typen unterscheiden:

\begin{tabular}{|c|c|c|c|}
\hline \multirow{7}{*}{$\begin{array}{l}\text { Bricolage- } \\
\text { Typen } \\
\text { nach } \\
\text { Ressourcen }\end{array}$} & & $\begin{array}{l}\text { Ressourcen/ } \\
\text { Sprachliche Einheit }\end{array}$ & Ressourcen im Korpus \\
\hline & Lautliche Bricolagen & Einzelne Laute & $\begin{array}{l}\text { Ethnolektale, lokal- } \\
\text { dialektale, gruppenspezi- } \\
\text { fische Laute }\end{array}$ \\
\hline & $\begin{array}{c}\text { Lexikalische Bricolagen: } \\
\text { Morphologische } \\
\text { Bricolagen } \\
\text { Semantische } \\
\text { Bricolagen }\end{array}$ & $\begin{array}{l}\text { Morpheme und Wortbe- } \\
\text { deutungen }\end{array}$ & $\begin{array}{l}\text { Dialektale, hochdeutsche, } \\
\text { englische Morpheme und } \\
\text { Begriffe sowie eigene } \\
\text { Neubildungen }\end{array}$ \\
\hline & $\begin{array}{l}\text { Phraseologische } \\
\text { Bricolagen }\end{array}$ & $\begin{array}{l}\text { Feste Wendungen/ Phra- } \\
\text { seologismen }\end{array}$ & $\begin{array}{l}\text { v. a. Schweizerdeutsche } \\
\text { Wendun- } \\
\text { gen/Phraseologismen }\end{array}$ \\
\hline & $\begin{array}{l}\text { Sprechstilistische } \\
\text { Bricolagen }\end{array}$ & $\begin{array}{l}\text { Sprechstile, Dialekte, } \\
\text { Sprachen, Spiel mit } \\
\text { eigener Mehrsprachig- } \\
\text { keit } \\
\text { Spiel mit fremden } \\
\text { Stimmen }\end{array}$ & $\begin{array}{l}\text { Stilisierungen u. a. von } \\
\text { lokalem Dialekt, ethno- } \\
\text { lektalem Stil } \\
\text { verschiedene Fremdspra- } \\
\text { chen }\end{array}$ \\
\hline & Intertextuelle Bricolagen & $\begin{array}{l}\text { Texte und Texte im } \\
\text { weiten Sinn (wie verfes- } \\
\text { tigte Gespräche und } \\
\text { Handlungsmus- } \\
\text { ter/Genres) }\end{array}$ & $\begin{array}{l}\text { Zeitungsbericht, Brief, } \\
\text { Liedtext, Witze, Comedy- } \\
\text { Beiträge, Verkaufsge- } \\
\text { spräch, Polizeirapport in } \\
\text { unterschiedlichen Dialek- } \\
\text { ten und Sprachen }\end{array}$ \\
\hline & *Ideelle Bricolagen 6 & $\begin{array}{l}\text { Gedanken, Ideen } \\
\text { (nicht primär sprachli- } \\
\text { che Ressourcen) }\end{array}$ & Erfundene Objekte \\
\hline
\end{tabular}

Tabelle 5: Bricolage-Typen nach Ressourcen (vgl. Galliker 2014: 449)

\subsubsection{Typisierung von Bricolagen nach Komplexitätsgrad: von minimal bis erweitert}

Wie anhand des Beispiels aufgezeigt wurde, können einzelne kleinere Bricolage-Schritte zu grösseren Bricolage-Sequenzen zusammengesetzt werden, d. h. Bricolagen können nach ihrem Komplexitätsgrad unterschieden werden. Die drei Typen „minimal“, "Normalfall“ und

\footnotetext{
6 Diese Ausprägung des Konzeptes wurde nur in Form eines Exkurses skizziert.
} 
„erweitert“ ergeben sich aus der Anzahl der beteiligten Sprechenden, der Anzahl Sequenzschritte, die sie aufweisen, sowie den verschiedenen Ressourcen und sprachlichen Ebenen, die gleichzeitig zum Einsatz kommen. Die Kategorisierung ist graduell und nicht trennscharf. Die folgende Übersicht soll dies verdeutlichen (cf. Galliker 2014: 382):

\begin{tabular}{|c|c|c|c|c|c|}
\hline \multirow{4}{*}{$\begin{array}{l}\text { Bricolage- } \\
\text { Typen } \\
\text { nach } \\
\text { Komplexi- } \\
\text { tätsgrad }\end{array}$} & \multirow{4}{*}{4} & \multirow[b]{2}{*}{$\begin{array}{l}\text { Minimale } \\
\text { Bricolagen }\end{array}$} & Beschreibung & $\begin{array}{l}\text { Verwendete } \\
\text { Verfahren }\end{array}$ & $\begin{array}{l}\text { Ressourcen/ } \\
\text { Sprachliche Ebene }\end{array}$ \\
\hline & & & $\begin{array}{l}\text { Kurzschrift } \\
\text { (Gumperz 1994: } \\
\text { 624) des Verfah- } \\
\text { rens, einzelne } \\
\text { Sprechhandlung } \\
2 \text { Sprechende }\end{array}$ & $\begin{array}{l}\text { Meist nur Sequenz- } \\
\text { schritt der Trans- } \\
\text { formation sowie } \\
\text { minimale interakti- } \\
\text { onale Reaktion } \\
\text { (z.B. Lachen) }\end{array}$ & $\begin{array}{l}\text { Von Einzellaut bis kur- } \\
\text { zer sprechstilistischer } \\
\text { Sequenz oder kurzem } \\
\text { Textausschnitt auf einer } \\
\text { sprachlichen Ebene }\end{array}$ \\
\hline & & $\begin{array}{l}\text { Bricolagen } \\
\text { (Normall- } \\
\text { fall) }\end{array}$ & $\begin{array}{l}\text { Einfaches Ver- } \\
\text { fahrens- } \\
\text { muster/Genre } \\
\text { Min. } 2 \text { Sprechen- } \\
\text { de }\end{array}$ & $\begin{array}{l}\text { Vorgabe } \\
\text { Übernahme } \\
\text { Transformation/ } \\
\text { Parallelisierung } \\
\text { tlw. mehrfach wie- } \\
\text { derholt }\end{array}$ & $\begin{array}{l}\text { Von Einzellaut bis } \\
\text { sprechstilistischer Se- } \\
\text { quenz oder Textaus- } \\
\text { schnitt versch. sprachl. } \\
\text { Ressourcen meist auf } \\
\text { einer sprachlichen Ebe- } \\
\text { ne }\end{array}$ \\
\hline & & $\begin{array}{l}\text { Erweiterte } \\
\text { Bricolagen }\end{array}$ & $\begin{array}{l}\text { Komplexes Ver- } \\
\text { fahrens- } \\
\text { muster/Genre } \\
\text { oft mehr als } 2 \\
\text { Sprechende }\end{array}$ & $\begin{array}{l}\text { Vorgabe } \\
\text { Übernahme } \\
\text { Transformation/ } \\
\text { Parallelisierung } \\
\text { mehrfach wieder- } \\
\text { holt }\end{array}$ & $\begin{array}{l}\text { Von Einzellaut bis } \\
\text { sprechstil. Sequenz oder } \\
\text { Textausschnitt versch. } \\
\text { sprachl. Ressourcen, } \\
\text { gleichzeitige Realisie- } \\
\text { rung versch. Bricolage- } \\
\text { Typen auf mehreren } \\
\text { sprachl. Ebenen }\end{array}$ \\
\hline
\end{tabular}

Tabelle 6: Bricolage-Typen nach Komplexitätsgrad (vgl. Galliker 2014: 450)

Entsprechend dieser Kategorisierung ist die Bricolage-Sequenz im Beispiel eine erweiterte Bricolage: Drei Sprecher beteiligen sich daran und es werden gleichzeitig verschiedene Ressourcen auf diversen sprachlichen Ebenen kontrastiert und verfremdet. Es ist ein komplexes Verfahrensmuster, in welchem die kommunikativen Verfahren der Vorgabe, Übernahme und Transformation sowie der minimalen Bricolagen (wie in den Tabelle 4 und 5 beschrieben) miteinander kombiniert und zu einem ganzen kommunikativen Genre zusammengefügt werden.

\subsubsection{Weitere Kriterien zur Charakterisierung von Bricolagen}

Die Bricolagen lassen sich jedoch nicht nur bezüglich ihres Komplexitätsgrades unterscheiden, auch der Verfestigungs- und Verfremdungsgrad können unterschieden werden. Des Weiteren gibt es verschiedene Verfremdungsebene. Tabelle 7 fasst die zentralen Kriterien zusammen (cf. Galliker 2014: 384): 


\begin{tabular}{|c|c|c|c|}
\hline \multirow{5}{*}{$\begin{array}{l}\text { Weitere Kri- } \\
\text { terien zur } \\
\text { Beschrei- } \\
\text { bung von } \\
\text { Bricolagen }\end{array}$} & & Kriterien & Beschreibung \\
\hline & Verfestigungsgrad & $\begin{array}{l}\text { Transitorische } \\
\text { Ad-hoc- } \\
\text { Bildungen } \\
\qquad \\
\text { Verfestigte } \\
\text { Bricolage- } \\
\text { Muster }\end{array}$ & $\begin{array}{l}\text { Bricolagen weisen unterschiedliche } \\
\text { Grade der Routine und dadurch der } \\
\text { Verfestigung auf: sie bewegen sich auf } \\
\text { einem Kontinuum zwischen spontanen, } \\
\text { transitorischen Ad-hoc-Bildungen über } \\
\text { die Kombination teilweise verfestigter } \\
\text { Elemente mit neuen Varianten zu ziem- } \\
\text { lich verfestigten, immer wieder wie- } \\
\text { derholten Variationen in verfestigten } \\
\text { Bricolage-Mustern. }\end{array}$ \\
\hline & Verfremdungsgrad & mimetisch & $\begin{array}{l}\text { Der Transformationsprozess stellt die } \\
\text { zentrale Sprechhandlung in Bricolagen } \\
\text { dar. Der Verfremdungsgrad des sprach- } \\
\text { lichen Materials durch die Transforma- } \\
\text { tion kann vom praktisch mimetischen } \\
\text { Zitieren des Originals bis zum starken } \\
\text { verfremdenden und gar karikierenden }\end{array}$ \\
\hline & & karikierend & Zitieren reichen. \\
\hline & $\begin{array}{l}\text { Verfremdungsebe- } \\
\text { ne }\end{array}$ & $\begin{array}{l}\text { strukturell } \\
\text { propositional } \\
\text { konversationell }\end{array}$ & $\begin{array}{l}\text { Drei Verfremdungsebenen können in } \\
\text { Bezug auf den in Bricolagen zentralen } \\
\text { Transformationsprozess unterschieden } \\
\text { werden: die strukturelle (Form), die } \\
\text { propositionale (Inhalt) und die konver- } \\
\text { sationelle (Gesprächsablauf), je nach- } \\
\text { dem welche Ebene transformiert wird. } \\
\text { Oft ereignet sich die Transformation } \\
\text { jedoch gleichzeitig auf mehreren Ebe- } \\
\text { nen. }\end{array}$ \\
\hline
\end{tabular}

Tabelle 7: Weitere Kriterien zur Beschreibung von Bricolagen (cf. Galliker 2014: 450)

In Bezug auf das analysierte Beispiel zeigen sich die folgenden Eigenschaften als relevant: Die Jugendlichen verwenden bspw. das hier beschriebene Muster der lautlichen Verfremdung sehr häufig. Das heisst, verschiedentlich setzen sie ortsdialektale Diphthonge in Begriffe ein, in welchen sie lautgesetzlich eigentlich nicht so vorkommen (cf. tuiback und auch meittwuch (Mittwoch), luica (Luca) etc., Galliker 2014: 188-201). Diese Art der Transformation hat sich im Gruppenstil bereits verfestigt und produziert neue Varianten, welche ihrerseits immer wieder Verwendung finden und so Kernelemente des Gruppenstils darstellen. Transitorische Adhoc-Bildungen sind hingegen die lautliche Bricolage mit der Aussprache von Englisch/Deutsch thuupack (Z 04) die morphologische Neubildung zuuphack (Z 07) sowie die Stilbastelei mit 50 Cent ( $Z$ 09). Diese drei letzten Elemente sind typische Stilelemente des Gruppenstils rein über die Verwendung des Verfahrens der Bricolage. So lieben es viele der Gruppenmitglieder sprachliche Normen $\mathrm{zu}$ brechen, indem sie Wortneubildungen aus der Kombination unterschiedlicher sprachlicher Ressourcen kreieren. Verbindungen von dialektalen und standardsprachlichen oder auch fremdsprachigen Morphemen kommen häufig vor, 
jedoch nicht immer mit denselben sprachlichen Einheiten (cf. u. a. Galliker 2014: 219-227 und Galliker 2010).

In Bezug auf die Verfremdung zeichnet sich das hier im Fokus stehende Beispiel vor allem durch Verfremdungen der sprachlichen Struktur aus. Einzig die sprechstilistische Bastelei auf Z 0950 Cent ist nicht strukturell verändert und klingt eher wie ein mimetisches Zitat. Die Verfremdung geschieht hier auf konversationeller Ebene: mit dem letzten Turn wird die in $Z$ 04 angelegte stilistische Anspielung auf den Rapper 2Pac durch die Wiederholung des gleichen Stils ratifiziert. Der Sprecher zeigt dadurch, dass er erstens die Anspielung verstanden hat und zweitens in der Lage ist, einen passenden stilistischen und auch inhaltlichen Beitrag zur Bricolage zu leisten. Das heisst, er stellt dar, dass er die in der Gruppe verwendeten Ressourcen kennt und weiss, wie er diese im gruppenspezifischen Verfahren der Bricolage treffend einsetzen kann.

\section{$7 \quad$ Fazit: Bricolage als stilbildendes Genre von Jugendlichen?}

Im Folgenden werden die oben dargestellten Überlegungen in einen erweiterten Interpretationszusammenhang gestellt. Einerseits werden die Bedeutung und Funktionen der Bricolagen für die untersuchte Peergruppe und ihren kommunikativen sozialen Stil verdeutlicht. Andererseits werden Verbindungen mit dem Makrokontext der Jugendsprachforschung aufgezeigt und ein Fazit gezogen zum konzeptuellen Zugang zur Erforschung von sprachlicher Variation.

\subsection{Bricolage als stilbildendes Genre eines kommunikativen sozialen Stils}

Im oben aufgeführten Beispiel passiert Folgendes: Drei Jugendliche transformieren ihre eigene unmarkierte sprachliche Variation (tuback) in eine englisch/deutsche Variante (thuuphack), die sie mit dem Kontext des US-amerikanischen Raps in Verbindung bringen. Gleichzeitig greifen sie auf lokalen Ortsdialekt zurück (tuiback), der kulturell und sozial in starkem Kontrast steht zur englischen Variante. Schliesslich integrieren sie ein hochdeutsches Morphem in die dialektale Lautumgebung (zuuphack). Sie bedienen sich also in einer sehr kurzen Sequenz der ganzen Breite ihres sprachlichen Repertoires - der inneren und äusseren Mehrsprachigkeit - um bestimmte kommunikative Ziele zu erreichen. Aus der neuartigen und eigenen Komposition dieser Varianten kreieren sie ihren gruppenspezifischen Stil, der eigenen sprachlichen Normen folgt. Dies ist jedoch nur erkennbar, wenn viele Gespräche der Jugendlichen analysiert werden und die Funktion ihres sprachlichen Verhaltens im erweiterten kommunikativen und sozialen Kontext betrachtet wird. Die folgenden Funktionen ihrer Sprechweise haben sich dabei als zentral erwiesen:

Funktionen auf der Mikroebene. Ausgehend von den sechs Funktionen der Sprache nach Hymes (1979: 67-69, Galliker 2014: 16-18) können auf der Mikroebene in diesem Beispiel gleichzeitig mehrere Funktionen der Bricolage festgestellt werden. Grundsätzlich steht am Anfang der Sequenz die direktive Funktion im Zentrum: David und Marco möchten beide gerne von den anderen den Tabak erhalten, um die Wasserpfeife aufzufüllen. Damit verbunden ist die Darstellungsfunktion: Es wird klargestellt, dass kein Tabak mehr da ist. Die Art und Weise, wie David diese Forderung jedoch gleich zu Beginn formuliert (thuuphack), aktiviert er sofort weitere Ebenen und Sprachfunktionen: Einerseits folgen sprachliche Basteleien 
auf lautlicher, morphologischer und sprechstilistischer Ebene, d. h. seine Formulierung gibt Anlass zu ästhetisierenden Reaktionen und hat somit eine poetische Funktion. Es wird viel sprachlicher Aufwand betrieben, nur um etwas Tabak zu erhalten. Andererseits wird ein Gruppenprozess angeregt, der bis zu einem gewissen Grad rivalisierend ist: Alle werden angespornt, einen eigenen Beitrag zur Formulierung zu leisten und ihr Können unter Beweis zu stellen. Hierbei geht es um die Aushandlung der persönlichen Position in der Gruppe, in welcher eine solche sprachliche Kompetenz hoch angesehen ist. Es handelt sich demzufolge um eine Form der Sprachprofilierung. Gleichzeitig ist es auch eine Form der gegenseitigen Bestätigung und Festigung der Gruppenidentität: Die Peers verstehen ihre Parallelisierungen, Kontrastierungen und Anspielungen gegenseitig, sie wissen, welche Ressourcen für solche Stilbasteleien in der Gruppe in Frage kommen und wie sie zu bewerten sind. In diesem Fall sind es Zugriffe auf dialektale und „globale“ (englische) Laute, deutsche Morpheme und einen stilisierten Sprechstil der amerikanischen Rap-Szene. Aus dieser Stilbastelei entsteht ein neuer eigener Stil, der sich gerade dadurch auszeichnet, eine Mischung verschiedener Stile und Stilelemente zu sein, und der von den beschriebenen Kontrastierungen lebt. Es handelt sich dabei um einen symmetrischen Prozess, da es den Beteiligten zwar um die eigene Sprachprofilierung geht und somit um die Positionierung in der Peergruppe (was teilweise asymmetrisch ist), jedoch grundsätzlich der gemeinsame kreative Akt im Vordergrund steht und die Gruppenidentität kontextualisiert wird.

Die ursprüngliche Bedeutung der verschiedenen Sprechstile des „urchigen“ (über tuiback) und des „Hip-Hop-Stils“ (über thuuphack) schwingt zwar in der Bricolage mit und ist Teil der Kontrastierungen, erhält aber im fortschreitenden Verfahren eine neue, eigene soziale Bedeutung als Teil des Gruppenstils. Genau dies ist typisch für Bricolagen: Stilelemente werden nicht nur zitiert, sondern erhalten, bezogen auf die Gruppe, eine neue eigene Bedeutung.

Funktionen auf der Mesoebene. Im Hinblick auf den sequenziellen Verlauf des gesamten Gesprächs unterstreicht diese Bricolage sprachlich den thematischen Wechsel von den Studierenden (resp. den Linguistinnen des Forschungsprojekts) zum aktuellen Geschehen - der Suche nach Tabak - und strukturiert somit den Diskurs. Funktional folgt auf eine Sequenz der Fremddarstellung eine Sequenz der Selbstdarstellung im Gruppenstil. Bereits in der Fremddarstellung machen sich die Gruppenmitglieder lustig über die „Studierenden/Forschenden“ (Z 01-03). Mit der anschliessenden Verdichtung von stilistischer Aktivität in der Bricolage grenzen sie sich klar von diesen ab und positionieren sich selber als Mitglieder ihrer eigenen Gruppe.

Auf diese Weise markieren die Jugendlichen ihre Gruppenzugehörigkeit beispielsweise auch im Beisein von Jugendlichen, die nicht zur Peergruppe gehören. Sie tun dies, indem sie schnell und routiniert auf die von der Gruppe präferierten Ressourcen zugreifen und im geeigneten Moment Bricolagen anreissen oder daran partizipieren. „Aussenstehenden“ Jugendlichen ist dies nicht im gleichen Masse möglich, da sie weder die passenden Ressourcen (insbesondere den lokalen Ortsdialekt) noch das Verfahrensmuster der Bricolage in gleicher Weise kennen und umsetzen können. Die anderen untersuchten Gruppen am Gymnasium verwenden Bricolagen kaum in vergleichbarem Ausmass in Bezug auf Quantität und Qualität. Legt man den Gruppenmitgliedern selber, aber auch aussenstehenden Jugendlichen, welche die Gruppenmitglieder kennen, solche Gesprächsausschnitte vor, sind diese in der Lage, Bricolagen als Verfahrensmuster resp. Genre zu erkennen und es als typisch für den Stil der Gruppe 
zu benennen. Die soziale Symbolisierung wird also aus emischer wie aus etischer Perspektive erkannt.

Funktionen auf Makroebene. In Bezug auf die Makroebene lässt sich folgendes beobachten: Die Jugendlichen spielen mit verschiedenen Aussprachevarianten eines Begriffs und stellen grundsätzlich zwei verschiedene Stile einander gegenüber: einerseits die übergeneralisierte lokale Dialektvariante (tuiback) und die auf die global verbreitete Jugendkultur des Hip-Hop anspielende ,englische“ Variante (thuuphack). Sie positionieren sich dadurch selber als ortskundige und lokal verbundene Jugendliche, die gleichzeitig Wissen über weiter verbreitete (jugendkulturelle) Trends aufweisen und diese gekonnt miteinander in Bezug setzen können. Durch die Verfremdung dieser Elemente in ihrem eigenen gemeinsamen Bricolage-Verfahren kreieren sie eine eigene (sprachliche) Wirklichkeit, mit einer eigenen Logik und eigenen Regeln, was zu einem Ausdruck der Andersartigkeit der eigenen Gruppe und ihres Verhaltens wird.

Nicht auf den ersten Blick ersichtlich anhand eines einzelnen Beispiels ist die Gewichtung und Bewertung der verwendeten stilistischen Ressourcen im Gros der Gruppenkommunikation. So verwenden die Jugendlichen zwar immer wieder stilistische Ressourcen einer globalen und auch medial verbreiteten Jugendkultur, wie z. B. Englisch oder ethnolektales Sprechen. Die lokalen Ressourcen - der traditionelle Ortsdialekt - werden von den Jugendlichen jedoch viel produktiver, häufiger und auch bevorzugt eingesetzt. Es scheint bei der untersuchten Gruppe gerade so zu sein, dass die starke Präsenz von medial verbreiteter „Jugendkultur“ und „Jugendsprache“ zu einer Rückbesinnung auf traditionelles und lokales Kulturgut führt. Trotzdem verwenden die Jugendlichen diese Ressourcen in einer distinkt anderen Weise, als dies ihre Eltern oder Schülerinnen und Schüler an ihrer Schule tun. Praktisch in jedem untersuchten Gespräch der Peers untereinander konnten Bricolagen ausgemacht werden, häufig sogar mehrere. In den Gesprächen anderer Jugendlicher an derselben Schule tauchten sie hingegen nur selten oder gar nicht auf (Galliker 2014: 403ff.).

Zusammenfassend lässt sich zu den Bricolagen sagen, dass sie in der untersuchten Peergruppe in besonderem Masse als stilbildendes Verfahrensmuster, also als kommunikatives Genre mit einer spezifischen sozialen Funktion eingesetzt werden. Bricolagen erweisen sich in der Gruppe als markierte Einheiten der Stilarbeit, durch das Grundprinzip der Inkorporierung neuen und fremden sprachlichen Materials. Auf der einen Seite sind die Bricolagen für die Gruppe also stilbildend, insofern als durch sie in musterhafter Weise mit Ressourcen umgegangen wird und den Ressourcen dadurch eine soziale Bedeutung zugeschrieben wird. Die Jugendlichen stellen sich durch die kompetente Umsetzung von und Teilnahme an Bricolagen als Mitglieder ihrer Peergruppe dar. Sie zeigen, dass sie die Regeln dieses Verfahrensmusters kennen und auch wissen, welche Ressourcen in welcher Weise dafür verwendet werden können.

Auf der anderen Seite entstehen innerhalb der Bricolagen viele verfestigte und routinierte Muster, die ihrerseits wieder als Schlüsselmerkmale des Gruppenstils weiterverwendet werden. Konkret heisst das, dass die Jugendlichen in ihrer Binnenkommunikation aus ihrem breiten Pool an sprachlichen Ressourcen Laute, Morpheme, Wörter und Wortbedeutungen, Phraseologismen und Satzteile sowie mehr oder weniger verfestigte kommunikative Stile, Gesprächs- und Textelemente auswählen und in eigener Weise zu einem Stil zusammensetzen. 
Sie gehen dabei nach dem bestimmten Muster der Bricolage vor und wenden konsequent das stilistische Prinzip des Kontrastierens an. Dabei verwenden und durchbrechen sie Regeln des Sprechens, die Formelhaftigkeit des Sprechens und spielen mit sprachlicher Variation. Wenn die Jugendlichen sprachliche Kontrastierungen vornehmen, geschieht dies jedoch nicht im Kontrast zu „einer“ Standardsprache oder Varietät. Kontrastiert wird in unterschiedliche Richtungen des Variationssystems und hintereinander in verschiedene Stillagen, Stile oder Sprachen. Dabei dient jeweils die direkt vorausgehende Variation als Referenz für die neue Variation. Das führt zu einer wahrnehmbaren dramaturgischen Steigerung innerhalb der Sequenzen und zum Phänomen des stilistischen Schillerns, also dem auffallenden und schnellen Wechsel zwischen verschiedenen Stilen und Stilelementen innerhalb von kurzen Gesprächssequenzen.

\subsection{Bricolage, sprachliche Kompetenz und Performanz}

Aus normativ-präskriptiver Sicht - also typischerweise aus der Sicht von Erwachsenen auf Jugendliches (Gesprächs-)Verhalten - werden im obigen Beispiel eine ganze Reihe von konversationellen Regeln und Normen gebrochen:

- Das „Prinzip der dialektalen Reinheit““ (cf. Haas 2004: 94), welches laut Haas in der Deutschschweiz vorherrscht und davon ausgeht, dass grundsätzlich in einem jeweils relativ einheitlichen Dialekt gesprochen wird und dieser nicht mit der Standardsprache oder anderen Varietäten vermischt wird.

- Die Kohärenz: es gibt klare inhaltliche Brüche zwischen Z 02 und 03 sowie 08 und 09 und die Funktion der Suche nach Tabak ist nur ansatzweise erkennbar.

- Die Kohäsion: tuback wird sehr oft in verschiedenen Varianten wiederholt, ohne eine klare Satzstruktur, und auch die grammatikalische Verbindung zwischen den einzelnen Gesprächsbeiträgen ist nur vage.

Es sind wohl genau solche Gespräche von Jugendlichen untereinander, die dazu führen, dass Erwachsene Jugendlichen sprachliche Kompetenz absprechen, oder welche in den Medien als „Sprachzerfall“ skandalisiert werden (cf. u. a. Guratzsch 2013, Neuland 2008: 3, Spitzmüller 2007).

Wie im oben angeführten Beispiel jedoch ersichtlich wird, zeigen die hier im Fokus stehenden Jugendlichen bei der Umsetzung von Bricolagen ein sehr hohes sprachliches Bewusstsein. Kern der Bricolagen ist die Sprechhandlung des Transformierens nach dem Stilprinzip des Kontrastierens. Ziel der Jugendlichen ist es, die einzelnen Gesprächsbeiträge der Teilnehmenden möglichst kontrastreich zu gestalten. Solche Kontraste werden v. a. durch die Kombination und Parallelisierung von stilistischen/sprachlichen Varianten erzeugt. Was die Jugendlichen also tun, ist, eine eigentliche rhetorische Strategie (cf. Gumperz 1994: 624) anzuwenden: Implizite oder explizite Regeln des Sprechens auf verschiedenen Ebenen des Ausdruckssystems sowie auch soziale und kulturelle Normen werden verletzt. Dies bedeutet aber auch, dass die Jugendlichen diese Regeln und Normen erkennen und anwenden können müssen, damit sie sie in Bricolagen brechen können.

Das sprachliche Bewusstsein der Jugendlichen, ihre Kenntnis sprachlicher, sozialer und kultureller Normen und insbesondere deren gezieltes Durchbrechen in den Bricolagen können als 
Ausdruck hoher sprachlicher Kompetenz eingeschätzt werden. Sie entsprechen Hymes' (1971, 1975) dreistufiger Hierarchisierung sprachlicher Kompetenz: dem Erkennen von sprachlichen Handlungsmustern, deren Reproduktion und schliesslich deren gültiger Umsetzung in der kommunikativen Praxis als performativer Akt. Die Art, wie die Jugendlichen Bricolagen in ihren Gesprächen umsetzen, stellt eine solche höchste Stufe der sprachlichen Kompetenz dar: Ein Vorführen sprachlicher und vor allem auch ästhetisierender Praktiken in einem kommunikativen Genre auf einer „Bühne“, nach spezifischen linguistischen, diskursiven und rhetorischen Prinzipien.

Wie aufgezeigt, werden Bricolagen funktional gezielt für die Binnenkommunikation und die Markierung von Gruppenzugehörigkeit eingesetzt. Wenn sich aussenstehende Sprechende daran stören, ist das ein Signal, dass diese soziale Positionierung gelingt und auch von aussen als solche wahrgenommen wird. Die Jugendlichen sind im Übrigen durchaus in der Lage, in anderen sozialen Kontexten und Gruppen andere kommunikative Stile einzusetzen.

\subsection{Bricolage in Beziehung und Abgrenzung zu anderen sprachlichen Verfahren}

Bricolagen, in der hier vorgestellten Form haben viel Ähnlichkeit mit und enthalten teilweise kommunikative Verfahren und Verfahrensmuster, welche v. a. für die Beschreibung der Kommunikation junger Gesellschaftsmitglieder eingesetzt werden. Für eine genaue Diskussion der Bezüge und Unterschiede vgl. Galliker (2014: 408-431):

- Konversationelles resp. Metaphorisches Code-Switching (Gumperz u. a. 1982, 1986), d. h. der sozial bedeutsame Wechsel zwischen verschiedenen Codes. Tritt als kommunikatives Verfahren in Bricolagen auf.

- Crossing (Rampton 1996, 1998), d. h. der sozial oder kulturell bedeutsame Wechsel in „fremde“ Codes und ,fremde Stimmen“ (u. a. auch Schwitalla 1994), tritt als Verfahren in Bricolagen auf.

- Polylanguaging (u. a. Jørgensen et al. 2011), d. h. die gleichzeitige Verwendung verschiedener Ressourcen mit eigener sozialer und kultureller Bedeutung. Tritt als Verfahren in Bricolagen auf.

- Bricolagen (u. a. Schlobinski et al. 1993, 2002), d. h. vornehmlich die Verwendung von medial verbreiteten kulturellen Ressourcen und Sprechstilen und deren Transformation für eigene Zwecke, können als spezifische Varianten (d. h. intertextuelle und sprechstilistische Bricolagen) des hier vorgestellten Bricolage-Konzeptes angesehen werden.

- Spiele mit Wortbildung, Wortbedeutung und Phraseologismen (u. a. Henne 1986, Androutsopoulos 1997), d. h. das Ausnützen insbesondere der strukturellen, aber auch der funktionalen Möglichkeiten, die in einer oder mehreren Sprachen zur Kreation und Ausweitung von Begriffen zur Verfügung stehen. Diese treten in Bricolagen häufig auf.

- Verschiedene für Jugendliche als typisch erachtete Genres wie die verbalen Duelle (Labov 1972), das Dissen (u. a. Deppermann/Schmidt 2001), das Lästern (Schubert 2009), das Frotzeln (u. a. Augenstein 1998, Schwitalla 1994), d. h. Verfahrensmuster, 
die sich durch bestimmte strukturelle und funktionale Charakteristika voneinander unterscheiden lassen, weisen Ähnlichkeiten mit Bricolage auf, legitimieren aber gleichzeitig Bricolage als Verfahrensmuster per se insbesondere über die für Bricolagen zentrale Funktion der Vergemeinschaftung.

Dieser erweiterte Kontext verdeutlicht, dass diese kommunikativen Verfahren und Verfahrensmuster und das in dieser Arbeit als Genre konzeptualisierte Verfahrensmuster der Bricolage im Sprechverhalten jugendlicher Gruppen offensichtlich stark verbreitete und verankerte Prinzipien darstellen und sich die Jugendlichen in ihrem sprachlichen Verhalten stark gleichen. Die genaue Umsetzung und Ausprägung der Muster und die entsprechenden kommunikativen Verfahren sowie die Regeln des Sprechens erweisen sich jedoch als stark kontextund netzwerkabhängig. D. h. innerhalb der untersuchten Netzwerke zeichnen sich lokale Eigenheiten in Bezug auf die verwendeten Ressourcen, deren soziale und kulturelle Bedeutung sowie auch deren Funktionen auf der Mikro-, Meso- und Makroebene der Gespräche ab und müssen von Gruppe zu Gruppe neu untersucht und beschrieben werden. ${ }^{7}$

\subsection{Makrokontext „Jugendsprache“"8 - Bricolage als typisch jugendliches Sprechver- halten?}

Die Frage, inwiefern sich Bricolagen als typisches Genre jugendlichen Sprechverhaltens ausweisen, kann kontrovers diskutiert werden. Wie oben aufgezeigt, kann über den Vergleich verschiedener Konzepte und Peergruppenuntersuchen verdeutlicht werden, dass es durchaus beachtliche Parallelen im sprachlichen Verhalten der Nidwaldner Peergruppe und diverser jugendlicher Gruppen in ganz Europa gibt. Verschiedene kommunikative Verfahren (wie Code-Switching, Polylanguaging, Crossing) und Verfahrensmuster (wie das Dissen, Frotzeln und Lästern) beschreiben sehr ähnliche sprachliche Handlungen in unterschiedlichen Kontexten. Sie lassen auf ähnliche kommunikative Verhaltensweisen von Jugendlichen schliessen, auch wenn die hier betrachteten Jugendlichen im Gegensatz zu den meisten anderen betrachteten Jugendgruppen aus einer ländlichen, „monolingualen“, primär dialektal sprechenden Umgebung stammen, praktisch ohne Berührungspunkte mit einem Migrationskontext, der in vielen Studien v. a. von Gruppen im urbanen Umfeld sehr zentral ist (cf. u. a. Jørgensen 2011 und Rampton 1996 und 1998).

Zudem scheint es so zu sein, dass gerade der Kontext von sozialen Netzwerken Jugendlicher, also die Peergruppen, das Auftreten von Bricolagen begünstigen, da ein ungezwungener Rahmen mit zeitlichem Überschuss ohne konkrete Aufgaben, engen sozialen Kontakten und geteiltem Hintergrundwissen ein idealer Ausgangspunkt für solche stilistischen Basteleien darstellt, zumal es deutlich mehr Zeit, Musse und Kreativität benötigt, in der oben beschriebenen Weise nach Tabak zu fragen, als mit einem einfachen Fragesatz. Was ebenfalls für die Interpretation von Bricolagen als typisch jugendliche Sprechweise spricht, ist, dass offensichtlich jüngere Mitglieder der erweiterten Sprechgemeinschaft die kommunikativen Praktiken

\footnotetext{
7 cf. z. B. auch die Bedeutung von dialektaler Variation für Wiener Jugendliche (Glauninger 2010).

8 Zur Diskussion des Begriffs „Jugendsprache“ cf. Galliker (2014: 25-26). Demzufolge wird hier nicht davon ausgegangen, dass es eine einzige Varietät „Jugendsprache“ gibt und alle Jugendlichen genau diese verwenden. Vielmehr wird analog zu Androutsopoulos (2006: 116) „Jugendsprache“ als abstrakte Varietät aufgefasst, die sich aus den Gemeinsamkeiten verschiedener Sprechstile jugendlicher Gruppen ergibt.
}

ISSN 1615-3014 
und insbesondere ihre Funktionen in den Bricolagen viel eher beschreiben und nachvollziehen können als Mitglieder älterer Generationen. So konnten z. B. ältere Studierende solche Bricolagen in Gesprächen als Einheiten erkennen und ihre Funktionen eruieren, sprachlich geschulte Lehrpersonen oder Eltern indes machten in den Gesprächen inhaltlich wie auch strukturell nur Nonsens aus.

Nicht zuletzt lassen sich auch aus psychologischer Sicht die zentralen Sprechhandlungen des Kontrastierens und Abweichens von sprachlichen, sozialen sowie kulturellen Normen und Regeln als typisches, transitorisches Element von Jugendlichkeit beschreiben (cf. Schäfers/Scherr 2005: 161 resp. Galliker 2014: 433-437).

Gleichwohl relativieren folgende Punkte die Interpretation von Bricolagen als ausschliesslich jugendtypisches Sprechverhalten: Da die Jugendlichen selber die Bricolagen hauptsächlich in ihrer Binnenkommunikation mit anderen Jugendlichen verwenden und nicht in Gesprächen mit Erwachsenen, markieren sie dadurch nicht direkt „Jugendlichkeit“" gegenüber älteren Gesellschaftsmitgliedern. Bei der Charakterisierung der Bricolagen als typisch jugendliches Sprechverhalten handelt es sich demnach eher um eine etische Zuschreibung einer Makrokategorie von „Jugend“ zu den sprachlichen Praktiken. Es zeigt sich zudem, dass bei Weitem nicht alle Jugendlichen Bricolagen als kommunikatives Verfahrensmuster in ihren Gesprächen verwenden - weder in den anderen untersuchten Gruppen aus der Schweiz noch in den entsprechenden Studien aus anderen Regionen. Die Häufigkeit und Ausprägungen des Auftretens von Bricolagen sind stark gruppenspezifisch und kontextabhängig und sie kommen durchaus auch in Gesprächen älterer Sprechender vor und auch dort nicht zwingend als Marker von Jugendlichkeit.

Bricolagen sind also nicht exklusiv jugendliche Sprechhandlungsmuster, kommen aber gehäuft in jugendlicher Binnenkommunikation in ganz Europa oder auch darüber hinaus vor. Blickt man über die spezifischen hier beschriebenen Prozesse hinaus, können Bricolagen auch als allgemein verbreitete Kulturtechnik betrachtet werden, die in vergemeinschaftender Funktion beispielsweise auch in Slam Poetry Battles oder Jazzimprovisationen zum Zuge kommt.

Festhalten lässt sich, dass sich Vergleiche des sprachlichen Handelns verschiedener sozialer Netzwerke, Peergruppen oder erweiterten Sprechgemeinschaften anstellen lassen, indem die detaillierte linguistische Beschreibung des Variationsraumes sowie der konzeptuelle und stilistische Zugang zu innerer und äusserer Mehrsprachigkeit miteinander kombiniert werden. Was die einzelnen sprachlichen Varianten und Variationen für die Peergruppe bedeuten und welche Bezüge dadurch sozial und kulturell hergestellt werden, versteht sich nur auf der Folie des beschriebenen Variationsraumes. Wie die Jugendlichen vom Lande jedoch mit ihren dialektalen und auch globalen Ressourcen als Gruppe umgehen, weist beachtliche Musterhaftigkeit und Parallelen zu vielen anderen (jugendlichen) Gruppen auf und nicht zuletzt zu einem Stil, der in vielen Studien eigentlich als „urban“ oder typisch für „Superdiversity“ bezeichnet wird (cf. Rampton 1998, Jørgensen 2011 und für eine erweiterte Darstellung Galliker (2014: 410-420). Es scheint ganz so, also ob die gleichzeitige Verwendung und eigenartige Kombination verschiedener sprachlicher Varianten, Stile oder Sprachen wie z. B. im Polylanguaging des late modern urban youth style in Norwegen (Jørgensen u. a. 2011) beschrieben oder wie in den Crossings in Englands von Migration geprägten Städten (Rampton u. a. 1998) eine recht verbreitete Kulturtechnik darstellt. Hier wurden sie über das Konzept der Bricolage 
gefasst und zwar für Jugendliche ohne Migrationshintergrund, aus einer ländlichen Alpenregion mit dialektaler Variation als Normallage und zentraler Ressource. Man könnte argumentieren, dass diese Parallelen zwischen Stadt und Land, zwischen In- und Ausland in Zeiten von Social Media nicht erstaunlich seien. Zum Zeitpunkt der Aufnahmen hatte allerdings noch keiner der Jugendlichen einen Social Media Account und manche noch nicht einmal eine eigene E-Mail-Adresse.

\section{Literaturverzeichnis}

Androutsopoulos, Jannis (1997): Deutsche Jugendsprache. Untersuchungen zu ihren Strukturen und Funktionen. Frankfurt a. M.: Lang. (=Nonstandard-Standard-Substandard 6).

Augenstein, Susanne (1998): Funktionen von Jugendsprache. Studien zu verschiedenen Gesprächstypen des Dialogs Jugendlicher mit Erwachsenen. Tübingen: Niemeyer. (= Reihe Germanistische Linguistik 192).

Clarke, John et al. (1979): Jugendkultur als Widerstand. Milieus, Rituale, Provokationen. Frankfurt a. M.: Syndikat.

Clarke, John et al. (1989) [1975]: "Style". In: Hall, Stuart/Jefferson, Tony (eds.): Resistance Through Rituals. Youth Subcultures in Post-war Britain. London, Unwin Hyman: 175191.

Coulmas, Florian (ed.) (1979): Dell Hymes: Soziolinguistik. Zur Ethnographie der Kommunikation. Frankfurt a. M., Suhrkamp: 29-270.

Denzin, Norman (1989) [1979]: The Research Act. A Theoretical Introduction to Sociological Methods. 3. Auflage. Englewood Cliffs/NJ: Prentice Hall.

Deppermann, Arnulf (2000): „Ethnographische Gesprächsanalyse: Zu Nutzen und Notwendigkeit von Ethnographie für die Konversationsanalyse“. Gesprächsforschung. OnlineZeitschrift zur verbalen Interaktion 1: 96-124.

Deppermann, Arnulf/Schmidt, Axel (2001): „Dissen: eine interaktive Praktik zur Verhandlung von Chrakter und Status in Peer-Groups männlicher Jugendlicher“". In: Sachweh, Svenja/Gessinger, Joachim (eds.)_Sprechalter. Duisburg, Red. OBST: 79-98. (=Osnabrücker Beiträge zur Sprachtheorie 62).

Galliker, Esther (2010): „Bricolage: Vom akkuraten Umgang Jugendlicher mit sprachlichen Ressourcen. Interlinguale und semantische Variation in Gesprächen Jugendlicher". In: Galliker, Esther / Kleinert, Andrea (eds.): Vielfalt der Empirie in der Angewandten Linguistik. Beiträge von Nachwuchsforschenden zu den 5. Tagen der Schweizer Linguistik. 20. bis 21. November 2008 am Departement Angewandte Linguistik der ZHAW Zürcher Hochschule für Angewandte Wissenschaften Winterthur. Hohengehren, Schneider: 31-48. (= Sprachenlernen Konkret! Angewandte Linguistik und Sprachvermittlung 10).

Galliker, Esther (2014): Bricolage. Ein kommunikatives Genre im Sprachgebrauch Jugendlicher aus der Deutschschweiz. Frankfurt a. M.: Peter Lang. (= Sprache - KommunikationKultur. Soziolinguistische Beiträge 14). doi:10.3726/978-3-653-03994-8.

Glauninger, Manfred Michael (2010): „Zwischen Hochdeutsch, Dialekt und Denglisch. ,Innere Mehrsprachigkeit' und urbane Kommunikation am Beispiel der Jugendlichen im Ballungsraum Wien.“ In: Gaisbauer, Stephan/Petermayr, Klaus (eds.): Übergang. Kommunikation in der Stadt und an ihren Rändern. Linz: Adalbert-Stifter-Inst. des Landes Oberösterreich. (= Schriften zur Literatur und Sprache in Oberösterreich 14). 
Gumperz, John (ed.) (1982a): Language and Social Identity. Cambridge: Cambridge University Press. (= Studies in Interactional Sociolinguistics 2).

Gumperz, John (ed.) (1982b): Discourse Strategies. Cambridge: Cambridge University Press. (= Studies in Interactional Sociolinguistics 1).

Gumperz, John (1992): “Contextualization Revisited.” In: Auer, Peter/Luzio di, Aldo (eds.): The Contextualization of Language. Amsterdam/Philadelphia, Benjamins: 39-54. (= Pragmatics \& Beyond New Series 22).

Gumperz, John (1994): „Sprachliche Variabilität in interaktionsanalytischer Perspektive“. In: Kallmeyer, Werner (ed.): Kommunikation in der Stadt. Exemplarische Analysen des Sprachverhaltens in Mannheim. Berlin/New York, de Gruyter: 612-639. (= Schriften des Instituts für Deutsche Sprache 4.1).

Gumperz, John/Hymes, Dell (eds.) (1986): Directions in Sociolinguistics. The Ethnography of Communication. 2. überarbeitete Auflage. Oxford: Blackwell.

Guratzsch, Dankwart (2013): „Das Gefühl des Sprachzerfalls trügt nicht“. Die Welt. www.welt.de/kultur/article114694278/Das-Gefuehl-des-Sprachverfalls-truegt-nicht.html. [14.07.2016].

Haas, Walter (2004): „Die Sprachsituation der deutschen Schweiz und das Konzept der Diglossie“. In: Christen, Helen (ed.): Dialekt, Regiolekt und Standardsprache im sozialen und zeitlichen Raum. Beiträge zum 1. Kongress der Internationalen Gesellschaft für Dialektologie des Deutschen, Marburg, Lahn, 5.-8. März 2003. Wien, Edition Praesens: 81110.

Henne, Helmut (1986): Jugend und ihre Sprache. Darstellung - Materialien - Kritik. Berlin/New York: de Gruyter.

Hymes, Dell (1971): “Competence and Performance in Linguistic Theory”. In: Huxley, Renira/Ingram, Elizabetz (eds.): Language Acquisition: Models and Methods. London, Academic Press: 3-28.

Hymes, Dell (1974) [2010]: Foundations in Sociolinguistics. An Ethnographic Approach. Philadelphia: University of Pennsylvania Press.

Hymes, Dell (1975): "Breakthrough into Performance". In: Ben-Amos, Dan/Goldstein, Kenneth S. (eds.): Folklore, Performance and Communication. The Hague, Mouton de Gruyter: $11-74$.

Jørgensen, Normann et al. (2011): "Polylanguaging in Superdiversity." Diversities 13/2: 2337.

Kallmeyer, Werner (ed.) (1994): Kommunikation in der Stadt. Bd. 1: Exemplarische Analysen des Sprachverhaltens in Mannheim. Berlin/New York: de Gruyter. (= Schriften des Instituts für Deutsche Sprache 4.1).

Kallmeyer, Werner (1995): „Zur Darstellung vom kommunikativem sozialem Stil in soziolinguistischen Gruppenporträts“. In: Keim, Inken/Kallmeyer, Werner (eds.): Kommunikation in der Stadt. Bd. 3: Kommunikative Stilistik einer sozialen Welt „kleiner Leute“ in der Mannheimer Innenstadt. Berlin/New York, de Gruyter: 1-39. (= Schriften des Instituts für deutsche Sprache 4.3).

Kallmeyer, Werner/Keim, Imken (2002): „Eigenschaften von sozialen Stilen der Kommunikation. Am Beispiel einer türkischen Migrantinnengruppe“. Osnabrücke Beiträge zur Sprachtheorie 65: 35-56. 
Kallmeyer, Wernerm/Keim, Imken (2003): "Linguistic Variation and the Construction of Social Identity in a German-Turkish setting. A case study of an immigrant youth group in Mannheim, Germany". In: Androutsopoulos, Jannis K./Georgakopoulou, Alexandra (eds.): Discourse Construction of Youth Identities. Amsterdam, Philadelphia: 27-46. (= Pragmatics and Beyond New Series 110).

Labov, William (1972): "Rules for Ritual Insults". In: Labov, William (ed.): Language in the Inner City. Philadelphia/PA, University of Pennsylvania Press: 297-353.

Lévi-Strauss, Claude (1963): Totemism. Boston: Beacon Press.

Lustat (2011): „Anteil der ausländischen Bevölkerung an der Gesamtbevölkerung. Europäische Städte“. Lustat. Statistik Luzern. www.lustat.ch/indikatoren/staedtevergleich/ bevoelkerung/auslaenderanteil. [13.07.2017].

Neuland, Eva (2001): „Subkulturelle Stile. Tendenzen der Substandardisierung in der deutschen Gegenwartssprache am Beispiel der Quersprachigkeit von Jugendlichen“. In: List, Gundula/List, Günther (eds.): Quersprachigkeit. Zum transkulturellen Registergebrauch in Laut- und Gebärdensprachen. Tübingen, Stauffenburg: 201-217. (= Tertiärsprachen 5).

Neuland, Eva (2008): Jugendsprache. Tübingen/Basel: Francke.

Pike, Kenneth Lee (1964): Language in Relation to a Unified Theory of the Structure of Human Behaviour. 2. Auflage. Den Haag: Mouton de Gruyter.

Rampton, Ben (1996): “Crossing: Language across Ethnic Boundaries. In: Coleman, Hywel/Cameron, Lynne (eds.): Change and Language. Papers from the Annual Meeting of the British Association for Applied Linguistics Held at the University of Leeds, September 1994. Clevedon, Multilingual Matters: 89-102.

Rampton, Ben (1998): "Language Crossing and the Redefinition of Reality". In: Auer, Peter (ed.): Code-Switching in Conversation. London, Routledge: 290-320.

Schäfers, Bernhard/Scherr, Albert (2005): Jugendsoziologie. Einführung in Grundlagen und Theorien. 8., umfassend aktualisierte und überarbeitete Auflage. Wiesbaden: Verlag für Sozialwissenschaften.

Schlobinski, Peter (2002): „Jugendsprache und Jugendkultur.“ Aus Politik und Zeitgeschichte. www.bpb.de/apuz/27135/jugendsprache-und-jugendkultur? $\mathrm{p}=$ all. [10.01.2016].

Schlobinski, Peter/Kohl, Gaby/Ludewigt, Irmgard (1993): Jugendsprache. Fiktion und Wirklichkeit. Opladen: Westdeutscher Verlag.

Schmid, Stephan/Tissot, Fabienne/Galliker, Esther (2011). „,S Beschte wos je hets gits“ oder wenn sich Schweizerdeutsch und Migrationssprachen treffen." Schweizerdeutsch. Zeitschrift für Sprache in der deutschen Schweiz 18/1: 11-14. www.ch-spraach.ch/ethnolekt. [10.01.2016].

Schubert, Daniel (2009): Lästern. Eine kommunikative Gattung des Alltags. Frankfurt a. M.: Lang. (= Sprache - Kommunikation - Kultur. Soziolinguistische Beiträge 6).

Schwitalla, Johannes (1994): „Die Vergegenwärtigung einer Gegenwelt. Sprachliche Formen der sozialen Abgrenzung einer Jugendgruppe in Vogelstang“. In: Kallmayer, Werner (ed.): Kommunikation in der Stadt. Bd. 1: Exemplarische Analysen des Sprachverhaltens in Mannheim. Berlin/New York, de Gruyter: 467-509. (= Schriften des Instituts für Deutsche Sprache 4.1).

Tissot, Fabienne/Schmid, Stephan/Galliker, Esther (2011): „Ethnolektales Schweizerdeutsch: soziophonetische und morphosyntaktische Merkmale sowie ihre dynamische Verwendung in ethnolektalen Sprechweisen“. In: Glaser, Elvira/Schmidt Jürgen Erich/Frey Natascha 
(eds.): Dynamik des Dialekts - Wandel und Variation. Akten des 3. Kongresses der Internationalen Gesellschaft für Dialektologie des Deutschen (IGDD). Stuttgart, Steiner: 319344. (= Zeitschrift für Dialektologie und Linguistik. Beihefte 144).

Werlen, Erika/Tissot, Fabienne/Galliker, Esther (2009): „Jugendsprache als Stil. Dialekt und Jugendsprache: Ethnographische Zugänge und Stil als sensitivierendes Konzept“". In: Henn-Memmesheimer, Beate/Franz, Joachim (eds.): Die Ordnung des Standard und die Differenzierung der Diskurse. Akten des 41. Linguistischen Kolloquiums in Mannheim 2006. Frankfurt a. M, Lang: 33-46.

Werlen, Erika/Galliker, Esther/Tissot, Fabienne (2010): „Konzeptuelle Zugänge zu intralingualer Variation: Dialekt und Standardsprache in Gesprächen Deutschschweizer Jugendlicher“‘. In: Jørgensen, Normann (ed.): Vallah, Gurkensalat $4 U$ \& Me! Current Perspectives in the Study of Youth Language. Frankfurt a. M., Lang: 229-244. (= Sprache - Kommunikation - Kultur. Soziolinguistische Beiträge 8). 